JOURNAL OF

SYMPLECTIC GEOMETRY

Volume 12, Number 3, 473-509, 2014

\title{
CONVERGENCE OF KÄHLER TO REAL POLARIZATIONS ON FLAG MANIFOLDS VIA TORIC DEGENERATIONS
}

\author{
Mark D. Hamilton and Hiroshi Konno
}

\begin{abstract}
In this paper, we construct a family of complex structures on a complex flag manifold that converge to the real polarization coming from the Gelfand-Cetlin integrable system, in the sense that holomorphic sections of a prequantum line bundle converge to delta-function sections supported on the Bohr-Sommerfeld fibers. Our construction is based on a toric degeneration of flag varieties and a deformation of Kähler structure on toric varieties by symplectic potentials.
\end{abstract}

\section{Introduction}

Let $(M, \omega)$ be a $2 n$-dimensional symplectic manifold. A prequantum line bundle $(L, h, \nabla)$ is a complex line bundle $L$ on $M$ with a Hermitian metric $h$ and a Hermitian connection $\nabla$, whose curvature equals $-2 \pi \sqrt{-1} \omega$. Geometric quantization is a procedure to assign a certain vector space, which is called a quantum Hilbert space, to $(M, \omega)$. To perform a geometric quantization procedure, we must choose a polarization, which is an integrable Lagrangian subbundle of the (complexification of the) tangent bundle $T M$ of $M$. Then, the quantum Hilbert space $\mathcal{H}(P)$ for a polarization $P$ is naively a subspace of (a certain completion of) the space of sections of $L$, consisting of covariantly constant sections along the polarization $P$. See $[\mathbf{W}]$ for general properties of geometric quantization.

The most common example of a polarization comes from an integrable complex structure $J$ on $M$ such that $(M, \omega, J)$ is a Kähler manifold. In this case, the anti-holomorphic tangent bundle $P_{J}=T^{0,1} M$ is a polarization, which we call a Kähler polarization. The quantum Hilbert space $\mathcal{H}\left(P_{J}\right)$ is the space of holomorphic sections $H^{0}\left(L, \bar{\partial}^{J}\right)$ with respect to the natural holomorphic structure $\bar{\partial}^{J}$ on $L$ induced by $J$. 
Another type of polarization, called a real polarization, is given by a foliation of $M$ into Lagrangian submanifolds. A completely integrable system $\mu: M \rightarrow \mathbb{R}^{n}$ (which is assumed to be proper) defines a singular real polarization $P_{\mu}$, where $\left(P_{\mu}\right)_{x}$ is the tangent space of the fiber of $\mu$ at each point $x \in M$. We set $B S(\mu)=\left\{p \in \mu(M) \mid H^{0}\left(\left.(L, h, \nabla)\right|_{\mu^{-1}(p)}\right) \neq 0\right\}$, where $H^{0}\left(\left.(L, h, \nabla)\right|_{\mu^{-1}(p)}\right)=\left\{s \in \Gamma\left(\left.(L, h, \nabla)\right|_{\mu^{-1}(p)}\right) \mid \nabla s=0\right\}$. Namely, $p \in B S(\mu)$ if and only if $\mu^{-1}(p)$ is a Bohr-Sommerfeld fiber. Then, the quantum Hilbert space $\mathcal{H}\left(P_{\mu}\right)$ is defined to be $\bigoplus_{p \in B S(\mu)} H^{0}\left(\left.(L, h, \nabla)\right|_{\mu^{-1}(p)}\right)[\mathbf{S}]$.

From the point of view of physics, the quantum Hilbert space should be independent of the choice of polarization. In particular, although Kähler and real polarizations seem to be quite different, the quantum Hilbert space for a Kähler polarization should be isomorphic to the one for a real polarization. There are several examples where this principle is observed to be true. A non-singular projective toric variety has a natural Kähler structure, and its moment map for the torus action induces a (singular) real polarization. It is well known that the dimension of the space of holomorphic sections of the prequantum line bundle is the number of lattice points in the image of the moment map, which is also the number of Bohr-Sommerfeld fibers in the variety. This implies that the principle holds in this case. In $[\mathbf{J W}]$, Jeffrey-Weitsman showed that the principle also holds in the case of the moduli space of flat connections over a compact Riemann surface.

A flag manifold with an integral symplectic structure has a singular real polarization defined by the Gelfand-Cetlin system, which was introduced by Guillemin-Sternberg in [GS], as well as a natural Kähler polarization since it is a complex manifold. In [GS], the authors studied the quantization of flag manifolds, and showed that the two polarizations give rise to quantizations with the same dimensions. However, their proof did not give any sort of direct relationship between the quantizations; rather, they computed the dimensions of the quantizations by other means (representation-theoretical and combinatoric) and showed they are equal.

One way of approaching the principle of independence of polarization is the following, considered by Baier, Florentino, Mourão and Nunes in $\left[\right.$ BFMN]. Fix a Kähler polarization $P_{J}$ and a real polarization $P_{\mu}$ on $(M, \omega)$. Then, the principle can be understood naturally if there is a family $\left\{P_{J_{s}}\right\}_{s \in[0, \infty)}$ of Kähler polarizations on $M$ with $P_{J_{0}}=P_{J}$ which converges to $P_{\mu}$ in the sense that there exists a basis $\left\{\sigma_{s}^{m}\right\}_{m \in B S\left(P_{\mu}\right)}$ of $\mathcal{H}\left(P_{J_{s}}\right)$ for each $s \in[0, \infty)$ such that, for each $m \in B S\left(P_{\mu}\right), \sigma_{s}^{m}$ converges to a deltafunction section supported on the Bohr-Sommerfeld fiber $\mu^{-1}(m)$ as $s$ goes to $\infty$. In $[$ BFMN], the authors carried out such a construction in the case of a non-singular projective toric variety by changing symplectic potentials, an important notion in the deformation theory of toric Kähler structures due to Guillemin $[\mathbf{G u 1 , ~ G u 2 ] ~ a n d ~ A b r e u ~ [ A b 1 , ~ A b 2 ] . ~}$ 
In this paper, we construct a family of Kähler polarizations on a flag manifold that converge to the real polarization coming from the GelfandCetlin system. See Theorem 2.1 for details. In doing so, we provide a direct relationship between the two quantizations. Our construction is based on the construction due to $[\mathbf{B F M N}]$ and the toric degeneration of a flag variety due to Kogan and Miller $[\mathbf{K M}]$. Originally, a toric degeneration of a flag variety was constructed in terms of representation theory $[\mathbf{G L}, \mathbf{C}]$. Later Kogan and Miller introduced deformed actions of a Borel subgroup on the space of matrices and described a toric degeneration of a flag variety explicitly. Moreover, they constructed a "degeneration in stages" of a flag variety to study the geometric meaning of the Gelfand-Cetlin basis of the irreducible representation of the unitary group. In [NNU], Nishinou, Nohara and Ueda pointed out that through the degeneration in stages one can identify the Gelfand-Cetlin system on the flag manifold with the integrable system on the limiting toric variety.

Our construction of a family of Kähler polarizations on a flag manifold proceeds as follows. We start from a flag manifold $F l_{n}$ embedded in the product of projective spaces $\mathbb{P}=\prod_{l=1, \ldots, n-1} \mathbb{P}\left(\bigwedge^{l} \mathbb{C}^{n}\right)$. For each $\left(a_{1}, \ldots, a_{n-1}\right) \in\left(\mathbb{Z}_{>0}\right)^{n-1}$, we fix a prequantum line bundle on $\mathbb{P}$ inducing a natural symplectic structure on $F l_{n}$. The toric degeneration of the flag variety $F l_{n}$ due to $[\mathbf{K M}]$ is a family of complex subvarieties $\left\{V_{t}\right\}_{t \in \mathbb{C}}$ in $\mathbb{P}$, where $V_{1}=F l_{n}$ and $V_{0}$ is a toric variety. Since all $V_{t}$ are diffeomorphic to each other for $t \neq 0$, the family $\left\{V_{t}\right\}_{t \neq 0}$ can be considered as a family of Kähler structures on the flag manifold $F l_{n}$. On the other hand, there is a family of toric Kähler structures $\left\{V_{0, s}\right\}_{s \in[0, \infty)}$ on $V_{0}$ with $V_{0,0}=V_{0}$, as considered in [BFMN] (explained above). If we could identify $F l_{n}$ with $V_{0, s}$ as a symplectic manifold, we could pull back the complex structures on $V_{0, s}$ to $F l_{n}$. However, the toric variety $V_{0}$ is not diffeomorphic to the flag manifold $F l_{n}$.

Instead, we consider a space $V_{t}$, which is still diffeomorphic to the flag manifold $F l_{n}$ but also is an approximation to $V_{0}$. We show that the deformation $\left\{V_{0, s}\right\}_{s \in[0, \infty)}$ can be realized as the restriction of a deformation of the ambient toric variety $\mathbb{P}$. The deformation of the ambient space induces a family of Kähler structures $\left\{V_{t, s}\right\}_{s \in[0, \infty)}$ on $V_{t}$ with $V_{t, 0}=V_{t}$ for each $t \in \mathbb{C}$. We develop a method to identify $V_{t, s}$ with $V_{t, 0}=V_{t}$ as a symplectic manifold. Moreover, we identify $F l_{n}$ with $V_{t}$ as a symplectic manifold by using the gradient-Hamiltonian flow (a notion that is due to Ruan $[\mathbf{R}]$ ) along a path that is an approximation of the path for degeneration in stages. Hence, we can pull the complex structure of $V_{t, s}$ back to $F l_{n}$. We also lift this identification to the prequantum line bundle in order to pull back holomorphic sections. Thus, we have a family of complex structures on the flag manifold with a fixed symplectic structure and a family of sections of the 
prequantum line bundle on the flag manifold, which are holomorphic with respect to the corresponding complex structure. Moreover, we give a precise estimate of these holomorphic sections, which allows us to prove that the holomorphic sections converge to delta-function sections supported on the Bohr-Sommerfeld fibers if we perform these two types of deformations simultaneously in an appropriate way.

The content of this paper is organized as follows. In Section 2, we state our main result. We review the results on a toric degeneration of a flag variety in Section 3. Then, we recall the gradient-Hamiltonian flow and construct its lift to the line bundle in Section 4. In Section 5, we review toric Kähler structures of toric manifolds, in particular, their deformation due to [BFMN]. In Section 6, we develop a method to identify submanifolds under the deformation of toric Kähler structures of the ambient toric manifolds. We also give an estimate of the change of holomorphic sections under this deformation. In Section 7, we prove the main result, constructing a family of complex structures on the flag manifold, and proving that holomorphic sections converge to delta-function sections supported on Bohr-Sommerfeld fibers.

\section{Main results}

Let $G L_{n}$ and $B$ be the general linear group and its Borel subgroup consisting of upper triangular matrices with $\mathbb{C}$-coefficients, respectively. The flag manifold is defined to be the complex manifold $F l_{n}=G L_{n} / B$. Let $\Lambda_{n}$ be the set of increasing indexes $I=\left(i_{1}<\cdots<i_{l}\right)$ with $1 \leq i_{1}, i_{l} \leq n$. For $I=\left(i_{1}<\cdots<i_{l}\right) \in \Lambda_{n}$ and $V=\left(v_{i j}\right) \in G L_{n}$, we set $|I|=l$ and

$$
p_{I}(V)=\operatorname{det}\left(\begin{array}{ccc}
v_{i_{1} 1} & \ldots & v_{i_{1} l} \\
\vdots & \ddots & \vdots \\
v_{i_{l} 1} & \ldots & v_{i_{l} l}
\end{array}\right)
$$

Then, the Pluckker embedding

$$
\rho: F l_{n} \rightarrow \mathbb{P}=\prod_{l=1}^{n-1} \mathbb{P}\left(\bigwedge \mathbb{C}^{n}\right)
$$

is defined by $[V] \mapsto\left(\left[p_{I}(V) ;|I|=1\right], \ldots,\left[p_{I}(V) ;|I|=n-1\right]\right)$, where $\left[x_{I} ;|I|=\right.$ $l]$ is the homogeneous coordinate of $\mathbb{P}\left(\bigwedge^{l} \mathbb{C}^{n}\right)$. Since the left $U(n)$-action on $M_{n}(\mathbb{C})$ commutes with the right $B$-action on $M_{n}(\mathbb{C}), U(n)$ acts on $F l_{n}$ from the left.

Next, we define a holomorphic line bundle on $F l_{n}$ and a Hermitian connection on it. Let $H_{l}$ be the hyperplane bundle on $\mathbb{P}\left(\bigwedge^{l} \mathbb{C}^{n}\right)$. It has a natural Hermitian metric $h_{l}$ such that $\frac{\sqrt{-1}}{2 \pi} R^{\nabla^{l}}=\omega_{l}$, where $R^{\nabla^{l}}$ is the curvature of the Chern connection $\nabla_{l}$ for the Hermitian metric $h_{l}$ and $\omega_{l} \in \Omega^{2}\left(\mathbb{P}\left(\bigwedge^{l} \mathbb{C}^{n}\right)\right)$ is the Fubini-Study form. Let $\pi_{l}: \mathbb{P} \rightarrow \mathbb{P}\left(\bigwedge^{l} \mathbb{C}^{n}\right)$ be the projection. Fix 
$\mathbf{a}=\left(a_{1}, \ldots, a_{n-1}\right) \in\left(\mathbb{Z}_{>0}\right)^{n-1}$. Then, we define a Kähler form $\omega_{\mathbb{P}}$ and a prequantum line bundle $\left(L^{\mathbb{P}}, h^{\mathbb{P}}, \nabla^{\mathbb{P}}\right)$ on $\mathbb{P}$ by

$$
\omega_{\mathbb{P}}=\sum_{l=1}^{n-1} a_{l} \pi_{l}^{*} \omega_{l} \in \Omega^{2}(\mathbb{P}),\left(L^{\mathbb{P}}, h^{\mathbb{P}}, \nabla^{\mathbb{P}}\right)=\bigotimes_{l=1}^{n-1} \pi_{l}^{*}\left(H_{l}, h_{l}, \nabla^{l}\right)^{\otimes a_{l}} .
$$

Then, $\nabla^{\mathbb{P}}$ is the Chern connection of $\left(L^{\mathbb{P}}, h^{\mathbb{P}}\right)$ and satisfies $\frac{\sqrt{-1}}{2 \pi} R^{\nabla^{\mathbb{P}}}=\omega_{\mathbb{P}}$. We set $\left(L^{F l_{n}}, h^{F l_{n}}, \nabla^{F l_{n}}\right)=\rho^{*}\left(L^{\mathbb{P}}, h^{\mathbb{P}}, \nabla^{\mathbb{P}}\right)$, i.e., $L^{F l_{n}}$ is a holomorphic line bundle on $F l_{n}$ with a Hermitian metric $h^{F l_{n}}$ and the Chern connection $\nabla^{F l_{n}}$ whose first Chern form is $\rho^{*} \omega_{\mathbb{P}}$. The $U(n)$-action on $F l_{n}$ preserves $\rho^{*} \omega_{\mathbb{P}}$ with a moment map $\mu_{U(n)}: F l_{n} \rightarrow \mathfrak{u}(n)^{*}$.

Next, we recall a certain completely integrable system on $F l_{n}$. Consider $U(l)$ for $l=1, \ldots, n-1$ as a subgroup of $U(n)$ defined by

$$
U(l)=\left\{\left(\begin{array}{cc}
A & O_{l, n-l} \\
O_{n-l, l} & E_{n-l}
\end{array}\right) \in U(n)\right\}
$$

where $O_{l, n-l} \in M_{l, n-l}(\mathbb{C})$ and $O_{n-l, l} \in M_{n-l, l}(\mathbb{C})$ are the zero matrices, $E_{n-l} \in M_{n-l}(\mathbb{C})$ is the unit element, and $A \in M_{l}(\mathbb{C})$. Let $\iota_{l}^{*}: \mathfrak{u}(n)^{*} \rightarrow \mathfrak{u}(l)^{*}$ be the dual map of the inclusion $\iota_{l}: \mathfrak{u}(l) \rightarrow \mathfrak{u}(n)$. Define a map $\lambda_{l}^{j}: \mathfrak{u}(l) \rightarrow \mathbb{R}$ such that $\lambda_{l}^{1}(A) \geq \cdots \geq \lambda_{l}^{l}(A)$ are eigenvalues of $-\sqrt{-1} A$ for $A \in \mathfrak{u}(l)$. We identify $\mathfrak{u}(l)$ with $\mathfrak{u}(l)^{*}$ by the invariant inner product. In $[\mathbf{G S}]$, Guillemin and Sternberg proved that

$$
\mu_{\mathrm{GC}}=\left(\lambda_{l}^{j} \circ \iota_{l}^{*} \circ \mu_{U(n)} ; 1 \leq l \leq n-1,1 \leq j \leq l\right): F l_{n} \rightarrow \mathbb{R}^{d}
$$

is a completely integrable system, where $d=\frac{1}{2} \operatorname{dim}_{\mathbb{R}} F l_{n}=\frac{n(n-1)}{2}$. The completely integrable system $\mu_{\mathrm{GC}}: F l_{n} \rightarrow \mathbb{R}^{d}$ and its image $\Delta_{\mathrm{GC}}=\mu_{\mathrm{GC}}\left(F l_{n}\right) \subset$ $\mathbb{R}^{d}$ are called the Gelfand-Cetlin system and the Gelfand-Cetlin polytope, respectively. Note that $\mu_{\mathrm{GC}}: F l_{n} \rightarrow \mathbb{R}^{d}$ is a continuous map and that it is smooth on $\mu_{\mathrm{GC}}^{-1}\left(\operatorname{Int} \Delta_{\mathrm{GC}}\right)$, where $\operatorname{Int} \Delta_{\mathrm{GC}}$ is the interior of $\Delta_{\mathrm{GC}}$. Moreover, $\mu_{\mathrm{GC}}^{-1}(m)$ is a $d$-dimensional real torus for each $m \in \operatorname{Int} \Delta_{\mathrm{GC}}$. In $[\mathbf{G S}]$, Guillemin and Sternberg also proved that, for $m \in \operatorname{Int} \Delta_{\mathrm{GC}} \subset \mathbb{R}^{d}$, the fiber $\mu_{\mathrm{GC}}^{-1}(m)$ is a Bohr-Sommerfeld fiber if and only if $m \in \operatorname{Int} \Delta_{\mathrm{GC}} \cap \mathbb{Z}^{d}$ and that the number of the points $\Delta_{\mathrm{GC}} \cap \mathbb{Z}^{d}$ coincides with the dimension of the space of holomorphic sections $H^{0}\left(L^{F l_{n}}, \bar{\partial}^{F l_{n}}\right)$, where $\bar{\partial}^{F l_{n}}$ is the holomorphic structure on $L^{F l_{n}}$. Namely, the quantum Hilbert space for the Kähler polarization on $F l_{n}$ is isomorphic to the one for the real polarization $P_{\mu_{\mathrm{GC}}}$ coming from the Gelfand-Cetlin system $\mu_{\mathrm{GC}}$.

In this paper, we construct a family of complex structures $\left\{J_{s}\right\}_{s \in[0, \infty)}$ on $F l_{n}$ such that the family of Kähler polarizations $\left\{P_{J_{s}}\right\}_{s \in[0, \infty)}$ converges to the real polarization $P_{\mu_{\mathrm{GC}}}$ in the following sense. 
Theorem 2.1. Let $\mathbb{F}$ and $J_{\mathbb{F}}$ be the underlying $C^{\infty}$-manifold and the complex structure of $F l_{n}$, respectively. Set $\omega_{\mathbb{F}}=\rho^{*} \omega_{\mathbb{P}} \in \Omega^{2}(\mathbb{F})$ and $d=$ $\operatorname{dim}_{\mathbb{R}} \mathbb{F}=\frac{n(n-1)}{2}$. Let $\left(L^{\mathbb{F}}, h^{\mathbb{F}}, \nabla^{\mathbb{F}}\right)$ be the underlying $C^{\infty}$ line bundle of $\left(L^{F l_{n}}, h^{F l_{n}}, \nabla^{F l_{n}}\right)$. Then, there exists a one-parameter family of $\left\{J_{s}\right\}_{s \in[0, \infty)}$ of complex structures on $\mathbb{F}$ which satisfies the following:

(1) $J_{s}$ is continuous with respect to the parameter $s \in[0, \infty)$.

(2) $J_{0}=J_{\mathbb{F}}$.

(3) $\left(\mathbb{F}, \omega_{\mathbb{F}}, J_{s}\right)$ is a Kähler manifold for each $s \in[0, \infty)$. So, for each $s \in[0, \infty)$, the Hermitian line bundle $\left(L^{\mathbb{F}}, h^{\mathbb{F}}, \nabla^{\mathbb{F}}\right)$ induces the holomorphic structure $\bar{\partial}^{s}$ on $L^{\mathbb{F}}$.

(4) For each $s \in[0, \infty)$, there exists a basis $\left\{\sigma_{s}^{m} \mid m \in \Delta_{\mathrm{GC}} \cap \mathbb{Z}^{d}\right\}$ of the space of holomorphic sections $H^{0}\left(L^{\mathbb{F}}, \bar{\partial}^{s}\right)$ such that, for each $m \in \operatorname{Int} \Delta_{\mathrm{GC}} \cap \mathbb{Z}^{d}$, the section $\frac{\sigma_{s}^{m}}{\left\|\sigma_{s}^{m}\right\|_{L^{1}(\mathbb{F})}}$ converges to a delta-function section supported on the Bohr-Sommerfeld fiber $\mu_{\mathrm{GC}}^{-1}(m)$ in the following sense: there exists a covariantly constant section $\delta_{m}^{\mathbb{F}}$ of $\left.\left(L^{\mathbb{F}}, h^{\mathbb{F}}, \nabla^{\mathbb{F}}\right)\right|_{\mu_{\mathrm{GC}}^{-1}(m)}$ and a measure $d \theta_{m}$ on $\mu_{\mathrm{GC}}^{-1}(m)$ such that, for any smooth section $\phi$ of the dual line bundle $\left(L^{\mathbb{F}}\right)^{*}$, the following holds:

$$
\lim _{s \rightarrow \infty} \int_{\mathbb{F}}\left\langle\phi, \frac{\sigma_{s}^{m}}{\left\|\sigma_{s}^{m}\right\|_{L^{1}(\mathbb{F})}}\right\rangle \frac{\omega_{\mathbb{F}}^{d}}{d !}=\int_{\mu_{\mathrm{GC}}^{-1}(m)}\left\langle\phi, \delta_{m}^{\mathbb{F}}\right\rangle d \theta_{m}
$$

Remark 2.2. By a similar argument as in the proof of Theorem 2.1, we can also prove that the support of the section $\sigma_{s}^{m}$ converges to $\mu_{\mathrm{CG}}^{-1}(m)$ as $s \rightarrow \infty$ for any $m \in\left(\Delta_{\mathrm{GC}} \backslash \operatorname{Int} \Delta_{\mathrm{GC}}\right) \cap \mathbb{Z}^{d}$. However, we cannot prove that $\sigma_{s}^{m}$ converges to a delta-function section for $m \in\left(\Delta_{\mathrm{GC}} \backslash \operatorname{Int} \Delta_{\mathrm{GC}}\right) \cap \mathbb{Z}^{d}$, because we do not yet have a sufficient description of $\mu_{\mathrm{CG}}^{-1}(m)$.

\section{Toric degeneration of flag varieties}

In $[\mathbf{K M}]$, Kogan and Miller constructed a toric degeneration of a flag variety based on a deformed Borel action. They also introduced degeneration in stages of a flag variety. In this section, we review their construction and recall its symplectic geometric aspects due to Nishinou, Nohara and Ueda [NNU].

3.1. Deformed Borel action and toric degeneration. First, we define the right action $\bullet$ of the product group $\left(G L_{n}\right)^{n}$ on $M_{n}(\mathbb{C})$ by

$$
V \bullet g=\left(\begin{array}{c}
\mathbf{v}_{1} g_{1} \\
\vdots \\
\mathbf{v}_{n} g_{n}
\end{array}\right) \text { for } V=\left(\begin{array}{c}
\mathbf{v}_{1} \\
\vdots \\
\mathbf{v}_{n}
\end{array}\right) \in M_{n}(\mathbb{C}) \text { and } g=\left(g_{1}, \ldots, g_{n}\right) \in\left(G L_{n}\right)^{n}
$$


Set $M_{n}\left(\mathbb{C}^{\times}\right)=\left\{\left(a_{i j}\right) \in M_{n}(\mathbb{C}) \mid a_{i j} \neq 0\right.$ for $\left.i, j=1, \ldots, n\right\}$. Define a map $\iota: M_{n}\left(\mathbb{C}^{\times}\right) \rightarrow\left(G L_{n}\right)^{n}$ by

$$
\iota\left(\left(a_{i j}\right)\right)=\left(\left(\begin{array}{ccc}
a_{11} & & O \\
& \ddots & \\
O & & a_{1 n}
\end{array}\right), \ldots,\left(\begin{array}{ccc}
a_{n 1} & & O \\
& \ddots & \\
O & & a_{n n}
\end{array}\right)\right) .
$$

Note that $\iota\left(M_{n}\left(\mathbb{C}^{\times}\right)\right)$is the maximal torus of $\left(G L_{n}\right)^{n}$. We also set

$$
T_{\mathrm{GC}}=\left\{\iota\left(\left(\begin{array}{cccc}
1 & & & 1 \\
a_{21} & & \ddots & \\
\vdots & \ddots & & \\
a_{n 1} & \ldots & a_{n n-1} & 1
\end{array}\right)\right) \mid\left(\begin{array}{cccc}
1 & & & 1 \\
a_{21} & & \ddots & \\
\vdots & \ddots & \\
a_{n 1} & \ldots & a_{n n-1} 1
\end{array}\right) \in M_{n}\left(\mathbb{C}^{\times}\right)\right\} .
$$

We also define a $k$-dimensional algebraic subtorus $T_{\mathrm{GC}}^{(k)}$ of $T_{\mathrm{GC}}$ by

$$
T_{\mathrm{GC}}^{(k)}=\left\{\iota\left(\left(a_{i j}\right)\right) \mid\left(a_{i j}\right) \in M_{n}\left(\mathbb{C}^{\times}\right), \quad i=k+1 \text { and } j \leq k \text { if } a_{i j} \neq 1\right\} .
$$

Then, we have

$$
T_{\mathrm{GC}}=\{1\} \times T_{\mathrm{GC}}^{(1)} \times \cdots \times T_{\mathrm{GC}}^{(n-2)} \times T_{\mathrm{GC}}^{(n-1)} .
$$

Next, we define the deformed Borel action as follows. For $t \in \mathbb{C}^{\times}$, we define $t^{\omega} \in M_{n}\left(\mathbb{C}^{\times}\right)$by

$$
\left(t^{\omega}\right)_{i j}=t^{\omega_{i j}}, \text { where } \omega_{i j}= \begin{cases}3^{i-j-1} & \text { if } i>j \\ 0 & \text { if } i \leq j\end{cases}
$$

In the above $\left(t^{\omega}\right)_{i j}$ is the $(i, j)$-component of $t^{\omega} \in M_{n}\left(\mathbb{C}^{\times}\right)$. Then, we define the deformed action $\bullet_{t}$ of $B$ on $M_{n}(\mathbb{C})$ by

$$
V \bullet t b=V \bullet\left\{\iota\left(t^{\omega}\right)(b, \ldots, b)\left(\iota\left(t^{\omega}\right)\right)^{-1}\right\},
$$

where $\iota\left(t^{\omega}\right),(b, \ldots, b), \iota\left(t^{\omega}\right)^{-1} \in\left(G L_{n}\right)^{n}$.

Let $\mathbb{C}\left[v_{i j} \mid 1 \leq i, j \leq n\right]$ be the coordinate ring of $M_{n}(\mathbb{C})$. Let $U \subset B$ the subgroup consisting of the matrices with 1 's on the diagonal. Then, the ring of $U$-invariant functions $\mathbb{C}\left[v_{i j} \mid 1 \leq i, j \leq n\right]^{U}$ for the deformed action $\bullet_{t}$ of $U$ is generated by the deformed Pluc̈ker coordinates

$$
\left\{q_{I}(V, t)=d_{I}\left(t^{\omega}\right)^{-1} p_{I}\left(V \bullet \iota\left(t^{\omega}\right)\right) \mid I \in \Lambda_{n}\right\}, \text { where } d_{I}\left(t^{\omega}\right)=\prod_{k=1}^{|I|}\left(t^{\omega}\right)_{i_{k} k}
$$

From the definition of $\omega \in M_{n}(\mathbb{Z})$, we see that $q_{I}(V, t)$ is a polynomial of $v_{i j}(1 \leq i, j \leq n)$ and $t$. Moreover, the deformed action $\bullet_{t}$ can be naturally extended to the case $t=0$. Thus, we have a quotient $F l_{n}(t)=M_{n}(\mathbb{C}) / /{ }_{t} B$ for all $t \in \mathbb{C}$, where the right-hand side is a GIT quotient by the deformed action $\bullet_{t}$. We also have a family $f:\left(M_{n}(\mathbb{C}) \times \mathbb{C}\right) / / B \rightarrow \mathbb{C}$ with $f^{-1}(t)=$ $F l_{n}(t) . F l_{n}(1)$ is nothing but the flag variety $F l_{n}$. Note that each $F l_{n}(t)$ is embedded in $\mathbb{P}$ by the deformed Pluc̈ker embedding $\rho_{t}: F l_{n}(t) \rightarrow \mathbb{P}$, which is defined by $[V] \mapsto\left(\left[q_{I}(V, t) ;|I|=1\right], \ldots,\left[q_{I}(V, t) ;|I|=n-1\right]\right)$ as in the 
case of the usual Pluc̈ker embedding. In $[\mathbf{K M}]$, Kogan and Miller proved the following, based on the argument in $[\mathbf{G L}]$.

Proposition 3.1. (1) The family $f:\left(M_{n}(\mathbb{C}) \times \mathbb{C}\right) / / B \rightarrow \mathbb{C}$ is flat.

(2) $F l_{n}(t)$ is biholomorphic to $F l_{n}$ for any $t \in \mathbb{C}^{\times}$. Moreover, $F l_{n}(0)$ is a toric variety on which the torus $T_{\mathrm{GC}}$ acts with an open dense orbit.

Let us give a few remarks about Proposition 3.1. Note that, if we set

$$
G L_{n}(t)=\left\{V \in M_{n}(\mathbb{C}) \mid V \bullet \iota\left(t^{\omega}\right) \in G L_{n}\right\},
$$

then we have $F l_{n}(t)=G L_{n}(t) /{ }_{t} B$ for $t \in \mathbb{C}^{\times}$, where the right-hand side is a geometric quotient of $G L_{n}(t)$ by the deformed action $\bullet_{t}$ of the Borel subgroup $B$. So, we see that $F l_{n}(t)$ is biholomorphic to $F l_{n}$ for any $t \in \mathbb{C}^{\times}$. Moreover, since the action $\bullet g$ on $M_{n}(\mathbb{C})$ for $g \in T_{\mathrm{GC}}$ commutes with the action $\bullet_{0} b$ on $M_{n}(\mathbb{C})$ for $b \in B$, the torus $T_{\mathrm{GC}}$ acts on $F l_{n}(0)=M_{n}(\mathbb{C}) / /_{0} B$. Thus, the family $f:\left(M_{n}(\mathbb{C}) \times \mathbb{C}\right) / / B \rightarrow \mathbb{C}$ can be viewed as a toric degeneration of a flag variety. The existence of a toric degeneration of a flag variety is originally proved in $[\mathbf{G L}, \mathbf{C}]$ in terms of representation theory. In $[\mathbf{A B}]$, a toric degeneration of a more general variety is constructed systematically.

3.2. Degeneration in stages. To relate the $U(n)$-action on $F l_{n}=F l_{n}(1)$ with the $T_{\mathrm{GC}}$-action on $F l_{n}(0)$, Kogan and Miller introduced degeneration in stages as follows. For $\tau=\left(t_{2}, \ldots, t_{n}\right) \in\left(\mathbb{C}^{\times}\right)^{n-1}$, we define $\tau^{\omega} \in M_{n}\left(\mathbb{C}^{\times}\right)$by

$$
\left(\tau^{\omega}\right)_{i j}=t_{i}^{\omega_{i j}} \text {, where } t_{1}=1 \text { and } \omega_{i j} \text { is given in (3.1). }
$$

In the above $\left(\tau^{\omega}\right)_{i j}$ is the $(i, j)$-component of $\tau^{\omega} \in M_{n}\left(\mathbb{C}^{\times}\right)$. Then, we define the deformed action $\bullet_{\tau}$ of $B$ on $M_{n}(\mathbb{C})$ by

$$
V \bullet \tau=V \bullet\left\{\iota\left(\tau^{\omega}\right)(b, \ldots, b)\left(\iota\left(\tau^{\omega}\right)\right)^{-1}\right\} .
$$

Thus, we have $F l_{n}(\tau)=M_{n}(\mathbb{C}) / / \tau B$ for $\tau \in\left(\mathbb{C}^{\times}\right)^{n-1}$ in the same way as in Section 3.1. We note that $F l_{n}(\tau)$ is also embedded in $\mathbb{P}$ by the deformed Plücker relations as $F l_{n}(t)$. Set

$$
\tau_{k}^{t}=(\underbrace{1, \ldots, 1}_{n-1-k}, t, \underbrace{0, \ldots, 0}_{k-1}) \in \mathbb{C}^{n-1} \text { for } t \in[0,1] \text { and } k=1, \ldots, n-1 .
$$

It is easy to see that $F l_{n}\left(\tau_{k}^{t}\right)=M_{n}(\mathbb{C}) / / \tau_{k}^{t} B$ is well defined. Note that $F l_{n}\left(\tau_{k}^{t}\right)$ has singularities if $\tau_{k}^{t}=\tau_{1}^{0}$ or $k \geq 2$. We call the family $\left\{F l_{n}\left(\tau_{k}^{t}\right)\right\}_{t \in[0,1]}$ the $k$ th stage of the degeneration. Note that

$$
\begin{aligned}
& U(n-k+1) \times T_{\mathrm{GC}}^{(n-1)} \times \cdots \times T_{\mathrm{GC}}^{(n-k+1)} \text { acts on } F l_{n}\left(\tau_{k}^{1}\right), \\
& U(n-k) \times T_{\mathrm{GC}}^{(n-1)} \times \cdots \times T_{\mathrm{GC}}^{(n-k+1)} \text { acts on } F l_{n}\left(\tau_{k}^{t}\right) \text { for } t \in(0,1), \\
& U(n-k) \times T_{\mathrm{GC}}^{(n-1)} \times \cdots \times T_{\mathrm{GC}}^{(n-k)} \text { acts on } F l_{n}\left(\tau_{k}^{0}\right),
\end{aligned}
$$


where $U(l)$ for $l=1, \ldots, n-1$ are the subgroups of $U(n)$ defined by $(2.1)$. Kogan and Miller considered the following degeneration in stages:

$$
\begin{gathered}
F l_{n}=F l_{n}\left(\tau_{1}^{1}\right) \stackrel{1 s t}{\longrightarrow} F l_{n}\left(\tau_{1}^{0}\right)=F l_{n}\left(\tau_{2}^{1}\right) \longrightarrow \cdots \longrightarrow F l_{n}\left(\tau_{k}^{1}\right) \stackrel{k t h}{\longrightarrow} F l_{n}\left(\tau_{k}^{0}\right) \\
\longrightarrow \cdots l_{n}\left(\tau_{n-1}^{0}\right)=F l_{n}(0) .
\end{gathered}
$$

In [NNU], Nishinou, Nohara and Ueda clarified the relation between the Gelfand-Cetlin system on the flag variety $F l_{n}$ and the completely integrable system on $F l_{n}(0)$ coming from its toric structure as follows. The smooth part $F l_{n}\left(\tau_{k}^{t}\right)^{\text {reg }}$ of $F l_{n}\left(\tau_{k}^{t}\right)$ has a symplectic structure $\iota_{\tau_{k}^{t}}^{*} \omega_{\mathbb{P}}$, where $\iota_{\tau_{k}^{t}}: F l_{n}\left(\tau_{k}^{t}\right)^{\mathrm{reg}} \rightarrow \mathbb{P}$ is the deformed Plücker embedding. Let $\mu_{U(n-k)}: F l_{n}\left(\tau_{k}^{t}\right)^{\text {reg }} \rightarrow \mathfrak{u}(n-k)$ be the moment map for $U(n-k)$-action on $F l_{n}\left(\tau_{k}^{t}\right)^{\text {reg }}$ for $t \in[0,1]$, where $\mathfrak{u}(n-k)$ is identified with $\mathfrak{u}(n-k)^{*}$ by the invariant inner product. Define a map $\lambda_{n-k}^{j}: \mathfrak{u}(n-k) \rightarrow \mathbb{R}$ such that $\lambda_{n-k}^{1}(A) \geq \cdots \geq \lambda_{n-k}^{n-k}(A)$ are eigenvalues of $-\sqrt{-1} A$ for $A \in \mathfrak{u}(n-k)$ as in Section 2. Then, in [NNU], the authors proved the following.

Proposition 3.2. There exists an open dense subset $F l_{n}\left(\tau_{k}^{t}\right)^{\circ} \subset F l_{n}\left(\tau_{k}^{t}\right)^{\mathrm{reg}}$ and a symplectic diffeomorphism $\varphi_{k}^{t_{2}, t_{1}}: F l_{n}\left(\tau_{k}^{t_{1}}\right)^{\circ} \rightarrow F l_{n}\left(\tau_{k}^{t_{2}}\right)^{\circ}$ for each $k=$ $1, \ldots, n-1, t \in[0,1]$ and $0 \leq t_{2} \leq t_{1} \leq 1$ which satisfy the following:

(1) $F l_{n}\left(\tau_{1}^{1}\right)^{\circ}=\mu_{\mathrm{GC}}^{-1}\left(\operatorname{Int} \Delta_{\mathrm{GC}}\right) \subset F l_{n}$ holds.

(2) $\varphi_{k}^{t, t}$ is the identity map for any $t \in[0,1]$. Moreover, $\varphi_{k}^{t_{3}, t_{2}} \circ \varphi_{k}^{t_{2}, t_{1}}=$ $\varphi_{k}^{t_{3}, t_{1}}$ holds for $0 \leq t_{3} \leq t_{2} \leq t_{1} \leq 1$.

(3) Under the identification of $F l_{n}\left(\tau_{k}^{t}\right)^{\circ}$ for all $t \in[0,1]$ by the maps $\varphi_{k}^{t_{2}, t_{1}}$, the moment map for $U(n-k) \times T_{\mathrm{GC}}^{(n-1)} \times \cdots \times T_{\mathrm{GC}}^{(n-k+1)}$-action on $F l_{n}\left(\tau_{k}^{t}\right)^{\circ}$ is independent of $t \in(0,1]$.

(4) $\left(\lambda_{n-k}^{j} \circ \mu_{U(n-k)} \mid 1 \leq j \leq n-k\right): F l_{n}\left(\tau_{k}^{0}\right)^{\circ} \rightarrow \mathbb{R}^{n-k}$ coincides with the moment map for the $T_{\mathrm{GC}}^{(n-k)}$-action on $F l_{n}\left(\tau_{k}^{0}\right)^{\circ}$.

The diffeomorphism $\varphi_{k}^{t_{2}, t_{1}}: F l_{n}\left(\tau_{k}^{t_{1}}\right)^{\circ} \rightarrow F l_{n}\left(\tau_{k}^{t_{2}}\right)^{\circ}$ is constructed by using the gradient-Hamiltonian flow due to Ruan $[\mathbf{R}]$, which is explained in the next section. The moment map for $U(n-k) \times T_{\mathrm{GC}}^{(n-1)} \times \cdots \times T_{\mathrm{GC}}^{(n-k+1)}$-action on $F l_{n}\left(\tau_{k}^{t}\right)^{\circ}$ induces the completely integrable system on $F l_{n}\left(\tau_{k}^{t}\right)^{\circ}$ in the same way as in the case of the Gelfand-Cetlin system. Proposition 3.2 implies the completely integrable system on $F l_{n}\left(\tau_{k}^{t}\right)^{\circ}$ for $t \in[0,1]$ and $1 \leq k \leq n-1$ remains the same during the degeneration in stages.

Due to Proposition 3.2, we have a diffeomorphism

$$
\Psi_{0}=\varphi_{n-1}^{0,1} \circ \varphi_{n-2}^{0,1} \circ \cdots \circ \varphi_{1}^{0,1}: F l_{n}^{\circ} \rightarrow F l_{n}(0)^{\circ}
$$


where $F l_{n}^{\circ}=F l_{n}\left(\tau_{1}^{1}\right)^{\circ}$ and $F l_{n}(0)^{\circ}=F l_{n}\left(\tau_{n-1}^{0}\right)^{\circ}$. Then, Nishinou, Nohara and Ueda proved the following.

Corollary 3.3. Let $\mu_{\mathrm{GC}}: F l_{n} \rightarrow \mathbb{R}^{\frac{n(n-1)}{2}}$ be the Gelfand-Cetlin system. Let $\mu_{T_{\mathrm{GC}}}: F l_{n}(0) \rightarrow\left(\mathfrak{t}_{\mathrm{GC}}\right)^{*}$ be the moment map for the action of $T_{\mathrm{GC}}$ on $F l_{n}(0)$. Then, there is a linear isomorphism $i: \mathbb{R}^{\frac{n(n-1)}{2}} \rightarrow\left(\mathfrak{t}_{\mathrm{GC}}\right)^{*}$ such that $i \circ \mu_{\mathrm{GC}}=$ $\mu_{T_{\mathrm{GC}}} \circ \Psi_{0}: F l_{n}^{\circ} \rightarrow\left(\mathfrak{t}_{\mathrm{GC}}\right)^{*}$. In particular, $F l_{n}(0)^{\circ}=\mu_{T_{\mathrm{GC}}}^{-1}\left(\operatorname{Int} \Delta_{\mathrm{GC}}\right) \subset F l_{n}(0)$ holds.

Therefore, the authors concluded that $F l_{n}(0)$ is a toric variety constructed from the Gelfand-Cetlin polytope $\Delta_{\mathrm{GC}}$. This fact is originally proved in $[\mathbf{K M}]$ in a different way. So, $F l_{n}(0)$ is called a Gelfand-Cetlin toric variety. Moreover, the Gelfand-Cetlin polytope $\Delta_{\mathrm{GC}}$ can be considered naturally as a subset of $\left(\mathfrak{t}_{\mathrm{GC}}\right)^{*}$. From now on, we consider the Gelfand-Cetlin system to be the map $\mu_{\mathrm{GC}}: F l_{n} \rightarrow\left(\mathfrak{t}_{\mathrm{GC}}\right)^{*}$.

\section{Gradient-Hamiltonian flow}

Let $(M, \omega, J)$ be a Kähler manifold. Let $f: M \rightarrow \mathbb{C}$ be a holomorphic function. Set $B=f(M)$ and $V_{c}=f^{-1}(c)$ for $c \in B$. Denote the inclusion map of $V_{c}$ by $\rho_{c}: V_{c} \rightarrow M$. Then, we have a family of symplectic manifolds $\left\{\left(V_{c}, \rho_{c}^{*} \omega\right)\right\}_{c \in B_{\text {reg }}}$ where $B_{\text {reg }}$ is the set of regular values of $f$. To identify these symplectic manifolds, Ruan introduced the gradient-Hamiltonian flow in $[\mathbf{R}]$. In this section, we recall the gradient-Hamiltonian flow and its basic properties. We also discuss the lift of the gradient-Hamiltonian flow to the prequantum line bundle.

By simple computations, we see that the following holds.

Lemma 4.1. Let $(M, \omega, J)$ be a Kähler manifold. Let $\Re f$ and $\Im f$ be the real and imaginary part of the holomorphic function $f: M \rightarrow \mathbb{C}$, respectively. Let $X_{\Im f} \in \mathcal{X}(M)$ be the Hamiltonian vector field of the function $\Im f$. Then, the following holds:

$$
X_{\Im f}=-\operatorname{grad}(\Re f) \text {, that } i s, i(-\operatorname{grad}(\Re f)) \omega=-d(\Im f) \text {, }
$$

where $i(-\operatorname{grad}(\Re f)) \omega$ is the contraction. In particular, $X_{\Im f}=-\operatorname{grad}(\Re f)$ is non-zero at a regular point of $f$.

Suppose that $f$ is proper and that each point in $M$ is a regular point of $f$. Then, we have the following vector field:

$$
Z=-\frac{\operatorname{grad}(\Re f)}{|\operatorname{grad}(\Re f)|^{2}}=\frac{X_{\Im f}}{\left|X_{\Im f}\right|^{2}} \in \mathcal{X}(M) .
$$


It is easy to see that

$$
Z(\Re f)=-1, \quad Z(\Im f)=0 \text { on } M .
$$

Since $f: M \rightarrow B$ is proper, for any $c \in B$ there exists $\epsilon_{c}>0$ such that the flow $\left\{\varphi_{t}\right\}_{t}$ generated by the vector field $Z \in \mathcal{X}(M)$ induces a diffeomorphism $\left.\varphi_{t}\right|_{V_{c}}: V_{c} \rightarrow V_{c-t}$ for $t \in\left(-\epsilon_{c}, \epsilon_{c}\right)$. In $[\mathbf{R}]$, Ruan found the following remarkable property.

Proposition 4.2. $\left(\left.\varphi_{t}\right|_{V_{c}}\right)^{*}\left(\rho_{c-t}^{*} \omega\right)=\rho_{c}^{*} \omega$ for $t \in\left(-\epsilon_{c}, \epsilon_{c}\right)$.

We call $Z \in \mathcal{X}(M)$ the gradient-Hamiltonian vector field and $\left\{\varphi_{t}\right\}_{t}$ the gradient-Hamiltonian flow, respectively.

Next, we discuss the lift of the gradient-Hamiltonian flow to the prequantum line bundle. Let us assume that there exists a prequantum line bundle $(L, h, \nabla)$ on $M$ in addition to the above setting. For any $c \in B$, we denote the restriction of $(L, h, \nabla)$ to the fiber $V_{c}$ by $\left(L^{V_{c}}, h^{V_{c}}, \nabla^{V_{c}}\right)$.

The horizontal lift $\tilde{Z} \in \mathcal{X}(L)$ of $Z \in \mathcal{X}(M)$ induces the flow $\left\{\tilde{\varphi}_{t}\right\}_{t}$, which is a lift of the gradient-Hamiltonian flow $\left\{\varphi_{t}\right\}_{t}$. Similarly, for any $c \in B$, there exists $\epsilon_{c}>0$ such that the flow $\left\{\tilde{\varphi}_{t}\right\}_{t}$ induces a bundle isomorphism $\left.\tilde{\varphi}_{t}\right|_{L^{V_{c}}}: L^{V_{c}} \rightarrow L^{V_{c-t}}$ for $t \in\left(-\epsilon_{c}, \epsilon_{c}\right)$.

Then, we have the following proposition. Since its proof does not seem to be found in the literature, we give a proof here.

Proposition 4.3. $\left(\left.\tilde{\varphi}_{t}\right|_{L^{V_{c}}}\right)^{*} \nabla^{V_{c-t}}=\nabla^{V_{c}}$ and $\left(\left.\tilde{\varphi}_{t}\right|_{L^{V_{c}}}\right)^{*} h^{V_{c-t}}=h^{V_{c}}$ for $t \in$ $\left(-\epsilon_{c}, \epsilon_{c}\right)$.

Proof. Since the connection $\nabla$ preserves the Hermitian metric $h$, the second assertion is obvious. So, we prove the first assertion.

Since $Z(\Im f)=0$ on $M$, the gradient-Hamiltonian flow $\left\{\varphi_{t}\right\}_{t}$ preserves $M_{\Im f=\Im c}=\{p \in M \mid \Im f(p)=\Im c\}$. First, we show that $i(Z) \omega=0$ on $M_{\Im f=\Im c}$. In fact, we have

$$
i(Z) \omega=i\left(\frac{X_{\Im f}}{\left|X_{\Im f}\right|^{2}}\right) \omega=\frac{-d(\Im f)}{\left|X_{\Im f}\right|^{2}}=0 \text { on } M_{\Im f=\Im c}
$$

Let $S \subset L$ be the unit sphere bundle and $p: S \rightarrow M$ the projection. If we denote the connection form of $\nabla$ by $\alpha \in \Omega^{1}(S)$, then we have $d \alpha=p^{*} \omega$. Since the restriction of the horizontal lift $\tilde{Z} \in \mathcal{X}(L)$ to $S$ can be considered as $\tilde{Z} \in \mathcal{X}(S)$, we have $i(\tilde{Z}) \alpha=0$ and $p_{*} \tilde{Z}=Z$. So, on $p^{-1}\left(M_{\Im f=\Im c}\right)$, we have

$$
L_{\tilde{Z}^{\alpha}}=i(\tilde{Z})\left(p^{*} \omega\right)=p^{*}\left\{i\left(p_{*} \tilde{Z}\right) \omega\right\}=p^{*}\{i(Z) \omega\}=0
$$


Thus, the flow induced by the vector field $\tilde{Z} \in \mathcal{X}(S)$ preserves the connection $\nabla$ on $p^{-1}\left(M_{\Im f=\Im c}\right)$.

\section{Toric Kähler structures of toric manifolds}

In this section, we review toric Kähler structures of toric manifolds. Starting from a Delzant polytope, we construct a symplectic toric manifold in Section 5.1 (due to $[\mathbf{D}]$, also described in $[\mathbf{G u 1}]$ ) and a complex toric manifold in Section 5.2 (described in $[\mathbf{A u}]$ and in Appendix 1 of $[\mathbf{G u 2}]$ ). See $[\mathbf{F}]$ for general properties of a complex toric manifold. We identify them according to a choice of symplectic potentials due to $[\mathbf{A b 1}, \mathbf{A b 2}, \mathbf{G u 1}, \mathbf{G u 2}]$ in Section 5.3. We also review a certain deformation of toric Kähler structures by changing symplectic potentials, which was introduced in [BFMN].

Let $T^{n}$ be a real torus with the Lie algebra $\mathfrak{t}^{n}$. Let

$$
\Delta=\left\{p \in\left(\mathfrak{t}^{n}\right)^{*} \mid\left\langle p, r_{j}\right\rangle+\lambda_{j} \geq 0 \text { for } j=1, \ldots, d\right\}
$$

be a bounded Delzant polytope, where $\langle\rangle:,\left(\mathfrak{t}^{n}\right)^{*} \times \mathfrak{t}^{n} \rightarrow \mathbb{R}$ is the natural pairing and $r_{j}$ is a primitive vector in the lattice $\mathfrak{t}_{\mathbb{Z}}^{n}$ for $j=1, \ldots, d$. We assume $\lambda_{1}, \ldots, \lambda_{d} \in \mathbb{Z}$. We set

$$
l_{j}(p)=\left\langle p, r_{j}\right\rangle+\lambda_{j}, F_{j}=\left\{p \in\left(\mathfrak{t}^{n}\right)^{*} \mid l_{j}(p)=0\right\} \text { for } j=1, \ldots, d .
$$

Let $T^{d}$ be a real torus with the Lie algebra $\mathfrak{t}^{d}$ and $X_{1}, \ldots, X_{d} \in \mathfrak{t}_{\mathbb{Z}}^{d}$ be the standard basis of $\mathfrak{t}^{d}$. Let $\pi: \mathfrak{t}^{d} \rightarrow \mathfrak{t}^{n}$ be the surjective Lie algebra homomorphism defined by $\pi\left(X_{j}\right)=r_{j}$ for $j=1, \ldots, d$. Then, the kernel of the corresponding Lie group homomorphism $\tilde{\pi}: T^{d} \rightarrow T^{n}$ is a connected subtorus $K$ of $T^{d}$ with the Lie algebra $\mathfrak{k}$. Let $u_{1}, \ldots, u_{d} \in\left(\mathfrak{t}^{d}\right)^{*}$ be the dual basis of $X_{1}, \ldots, X_{d} \in \mathfrak{t}_{\mathbb{Z}}^{d}$. We set $\lambda_{\Delta}=\lambda_{1} u_{1}+\cdots+\lambda_{d} u_{d} \in\left(\mathfrak{t}^{d}\right)_{\mathbb{Z}}^{*}$.

5.1. A symplectic toric manifold $\boldsymbol{M}_{\text {symp }}$. Let $\tilde{\omega}$ be the standard symplectic form on $\mathbb{C}^{d}$. The natural action of $T^{d}$ on $\left(\mathbb{C}^{d}, \tilde{\omega}\right)$ admits a moment map $\mu_{T^{d}}: \mathbb{C}^{d} \rightarrow\left(\mathfrak{t}^{d}\right)^{*}$, given by $\mu_{T^{d}}(z)=\pi \sum_{j=1}^{d}\left|z_{j}\right|^{2} u_{j}$, where $z=\left(z_{1}, \ldots, z_{d}\right)$. If we denote the dual map of the inclusion $\iota: \mathfrak{k} \rightarrow \mathfrak{t}^{d}$ by $\iota^{*}:\left(\mathfrak{t}^{d}\right)^{*} \rightarrow \mathfrak{k}^{*}$, then the moment map $\mu_{K}: \mathbb{C}^{d} \rightarrow \mathfrak{k}^{*}$ for the action of the subtorus $K$ on $\left(\mathbb{C}^{d}, \tilde{\omega}\right)$ is given by $\mu_{K}(z)=\pi \sum_{j=1}^{d}\left|z_{j}\right|^{2} \iota^{*} u_{j}$. The compact symplectic toric manifold $M_{\text {symp }}$ is defined to be the symplectic quotient $M_{\text {symp }}=\mu_{K}^{-1}\left(\iota^{*} \lambda_{\Delta}\right) / K$ with the natural symplectic structure $\omega \in \Omega^{2}\left(M_{\text {symp }}\right)$. The quotient torus $T^{n}=T^{d} / K$ acts on $\left(M_{\text {symp }}, \omega\right)$ with the moment map $\mu_{T^{n}}: M_{\text {symp }} \rightarrow\left(\mathfrak{t}^{n}\right)^{*}$. Since $\mu_{T^{d}}(z)-\lambda_{\Delta} \in \operatorname{ker}\left\{\iota^{*}:\left(\mathfrak{t}^{d}\right)^{*} \rightarrow\right.$ $\left.\mathfrak{k}^{*}\right\}=$ image $\left\{\pi^{*}:\left(\mathfrak{t}^{n}\right)^{*} \rightarrow\left(\mathfrak{t}^{d}\right)^{*}\right\}$, it is given by $\mu_{T^{n}}([z])=\left(\pi^{*}\right)^{-1}\left(\mu_{T^{d}}(z)-\right.$ $\left.\lambda_{\Delta}\right) \in\left(\mathfrak{t}^{n}\right)^{*}$. It is well known that $\mu_{T^{n}}\left(M_{\text {symp }}\right)=\Delta$. 
Next, we define a prequantum line bundle on $M_{\text {symp }}$. Let $\tilde{L}_{\text {symp }}=\mathbb{C}^{d} \times \mathbb{C}$ be the trivial line bundle with the standard fiber metric $\tilde{h}$. Let $\tilde{\nabla}$ be a Hermitian connection on $\tilde{L}_{\text {symp }}$ defined by $\tilde{\nabla}=d-\sqrt{-1} \pi \sum_{i=j}^{d}\left(x_{j} d y_{j}-y_{j} d x_{j}\right)$, where $x_{j}, y_{j}$ are the real and imaginary part of $z_{j}$, respectively. The action of $T^{d}$ on $\tilde{L}_{\text {symp }}$ defined by $(z, v) \operatorname{Exp}_{T^{d}} \xi=\left(z \operatorname{Exp}_{T^{d}} \xi, v e^{2 \pi \sqrt{-1}\left\langle\lambda_{\Delta}, \xi\right\rangle}\right)$ preserves the Hermitian metric $\tilde{h}$ and the connection $\tilde{\nabla}$, where $\operatorname{Exp}_{T^{d}}: \mathfrak{t}^{d} \rightarrow T^{d}$ is the exponential map. Then, the prequantum line bundle $\left(L_{\text {symp }}, h, \nabla\right)$ on $M_{\text {symp }}$ is defined to be the quotient of the restriction of $\tilde{L}_{\text {symp }}$ to $\mu_{K}^{-1}\left(\iota^{*} \lambda_{\Delta}\right)$ by the action of the subtorus $K$. Moreover, the quotient torus $T^{n}=T^{d} / K$ acts on $L_{\text {symp }}$, preserving $h$ and $\nabla$. Let $[z]_{K} \in M_{\text {symp }}$ denote a point represented by $z \in \mu_{K}^{-1}\left(\iota^{*} \lambda_{\Delta}\right)$. Similarly, $[z, v]_{K}$ denotes a point in $L_{\text {symp }}$ represented by $(z, v) \in \mu_{K}^{-1}\left(\iota^{*} \lambda_{\Delta}\right) \times \mathbb{C}$.

Set $M_{\text {symp }}^{0}=\mu_{T^{n}}^{-1}(\operatorname{Int} \Delta)$, where Int $\Delta$ is the interior of the Delzant polytope $\Delta$. Then, it is easy to see that $\left(\sqrt{\frac{l_{1}(p)}{\pi}}, \ldots, \sqrt{\frac{l_{d}(p)}{\pi}}\right) \in \mu_{K}^{-1}\left(\iota^{*} \lambda_{\Delta}\right)$ for any $p \in \operatorname{Int} \Delta$. Therefore, the map $\psi_{\text {symp }}^{0}: \operatorname{Int} \Delta \times \mathfrak{t}^{n} / \mathfrak{t}_{\mathbb{Z}}^{n} \rightarrow M_{\text {symp }}^{0}$ defined by

$$
\begin{aligned}
\psi_{\text {symp }}^{0}(p,[q]) & =\left[\left(\sqrt{\frac{l_{1}(p)}{\pi}}, \ldots, \sqrt{\frac{l_{d}(p)}{\pi}}\right)\right]_{K} \operatorname{Exp}_{T^{n}}(q) \\
& =\left[\left(\sqrt{\frac{l_{1}(p)}{\pi}} e^{2 \pi \sqrt{-1}\left\langle u_{1}, \tilde{q}\right\rangle}, \ldots, \sqrt{\frac{l_{d}(p)}{\pi}} e^{2 \pi \sqrt{-1}\left\langle u_{d}, \tilde{q}\right\rangle}\right)\right]_{K}
\end{aligned}
$$

is a diffeomorphism, where $\tilde{q} \in \mathfrak{t}^{d}$ is taken so that $\pi(\tilde{q})=q$. Note that we have

$$
\mu_{T^{n}} \circ \psi_{\text {symp }}^{0}(p,[q])=p \quad \text { for }(p,[q]) \in \operatorname{Int} \Delta \times \mathfrak{t}^{n} / \mathfrak{t}_{\mathbb{Z}}^{n}
$$

Next, we define a section $s_{\text {symp }}^{0}$ of $L_{\text {symp }}$ restricted to $M_{\text {symp }}^{0}$ by

$$
s_{\text {symp }}^{0}(p,[q])=\left[\left(\sqrt{\frac{l_{1}(p)}{\pi}}, \ldots, \sqrt{\frac{l_{d}(p)}{\pi}}\right), 1\right]_{K} \operatorname{Exp}_{T^{n}}(q) \in L_{\text {symp }} .
$$

This section induces a unitary trivialization of the prequantum line bundle $L_{\text {symp }}$ on $M_{\text {symp }}^{0}$.

Fix a $\mathbb{Z}$-basis $p_{1}, \ldots, p_{n} \in\left(\mathfrak{t}^{n}\right)_{\mathbb{Z}}^{*}$ and its dual basis $q_{1}, \ldots, q_{n} \in \mathfrak{t}_{\mathbb{Z}}^{n}$. Set $\Delta^{0}=$ $\left\{x=\left(x_{1}, \ldots, x_{n}\right) \in \mathbb{R}^{n} \mid \sum_{i=1}^{n} x_{i} p_{i} \in \operatorname{Int} \Delta\right\}$. Then, we have a coordinate $(x,[\theta]) \in \Delta^{0} \times \mathbb{R}^{n} / \mathbb{Z}^{n}$ on Int $\Delta \times \mathfrak{t}^{n} / \mathfrak{t}_{\mathbb{Z}}^{n}$. So, $(x,[\theta]) \in \Delta^{0} \times \mathbb{R}^{n} / \mathbb{Z}^{n}$ can be considered as a coordinate on $M_{\text {symp }}^{0}$. It is easy to see the following by simple computations.

Lemma 5.1. Let $(x,[\theta]) \in \Delta^{0} \times \mathbb{R}^{n} / \mathbb{Z}^{n}$ be the coordinate on $M_{\text {symp }}^{0}$ induced by the fixed basis $p_{1}, \ldots, p_{n} \in\left(\mathfrak{t}^{n}\right)_{\mathbb{Z}}^{*}$. Then, the symplectic form $\omega$ on $M_{\mathrm{symp}}^{0}$ 
and the connection $\nabla$ on $\left.L_{\mathrm{symp}}\right|_{M_{\mathrm{symp}}^{0}}$ are described as follows.

(1) $\left.\omega\right|_{M_{\mathrm{symp}}^{0}}=\sum_{i=1}^{n} d x_{i} \wedge d \theta_{i}$.

(2) $\left.\nabla\right|_{M_{\text {symp }}^{0}} ^{0}=d-2 \pi \sqrt{-1} \sum_{i=1}^{n} x_{i} d \theta_{i}$ with respect to the unitary trivialization defined by the section $s_{\text {symp }}^{0}$ on $M_{\text {symp }}^{0}$.

(3) For $m \in \operatorname{Int} \Delta, \mu_{T^{n}}^{-1}(m)$ is a Bohr-Sommerfeld fiber for the prequantum line bundle $\left(L_{\mathrm{symp}}, h, \nabla\right)$ if and only if $m \in \operatorname{Int} \Delta \cap\left(\mathfrak{t}^{n}\right)_{\mathbb{Z}}^{*}$. Moreover, $\delta_{m}([\theta])=\left.e^{2 \pi \sqrt{-1} \sum_{i=1}^{n} m_{i} \theta_{i}} s_{\mathrm{symp}}^{0}\right|_{\mu_{T^{n}}^{-1}(m)}$ is a covariantly constant section of $\left.\left(L_{\mathrm{symp}}, h, \nabla\right)\right|_{\mu_{T^{n}(m)}^{-1}}$ for $m=\sum_{i=1}^{n} m_{i} p_{i} \in \operatorname{Int} \Delta \cap\left(\mathfrak{t}^{n}\right)_{\mathbb{Z}}^{*}$, where $[\theta] \in \mathbb{R}^{n} / \mathbb{Z}^{n}$ is a coordinate on $\mu_{T^{n}}^{-1}(m)$.

5.2. A complex toric manifold $\boldsymbol{M}_{\text {comp }}$. Let $\Delta$ be a Delzant polytope defined by (5.1), and denote the set of its vertices by $\Delta(0)$. Let $F_{j} \subset\left(\mathfrak{t}^{n}\right)^{*}$ be the hyperplane defined in (5.2) for $j=1, \ldots, d$. For each $v \in \Delta(0)$, we set $\Lambda_{v}=\left\{j \mid v \in F_{j}\right\}, \mathbb{C}_{v}^{d}=\left\{z \in \mathbb{C}^{d} \mid z_{j} \neq 0\right.$ if $\left.j \in\{1, \ldots, d\} \backslash \Lambda_{v}\right\}$ and $\mathbb{C}_{\Delta}^{d}=\bigcup_{v \in \Delta(0)} \mathbb{C}_{v}^{d}$. Then, the compact complex toric manifold $M_{\text {comp }}$ is defined to be the quotient space $M_{\text {comp }}=\mathbb{C}_{\Delta}^{d} / K_{\mathbb{C}}$, where $K_{\mathbb{C}}$ is the complexification of the subtorus $K$. Similarly, the complexification of the torus $T^{d}$ is denoted by $T_{\mathbb{C}}^{d}$. The quotient torus $T_{\mathbb{C}}^{n}=T_{\mathbb{C}}^{d} / K_{\mathbb{C}}$ acts on $M_{\text {comp }}$, preserving its complex structure $J$.

Next, we define a holomorphic line bundle on $M_{\text {comp }}$. Let $\tilde{L}_{\text {comp }}=\mathbb{C}^{d} \times \mathbb{C}$ be a trivial holomorphic line bundle on $\mathbb{C}^{d}$. Define the action of $T_{\mathbb{C}}^{d}$ on $\tilde{L}_{\text {comp }}$ by $(z, v) \operatorname{Exp}_{T_{\mathbb{C}}^{d}} \xi=\left(z \operatorname{Exp}_{T_{\mathbb{C}}^{d}} \xi, v e^{2 \pi \sqrt{-1}\left\langle\lambda_{\Delta}, \xi\right\rangle}\right)$. The holomorphic line bundle $L_{\text {comp }}$ is defined to be the quotient of the restriction of $\tilde{L}_{\text {comp }}$ to $\mathbb{C}_{\Delta}^{d}$ by the action of $K_{\mathbb{C}}$. Then, the quotient torus $T_{\mathbb{C}}^{n}=T_{\mathbb{C}}^{d} / K_{\mathbb{C}}$ acts on $L_{\text {comp }}$, preserving its holomorphic structure $\bar{\partial}$. Let $[z]_{K_{\mathbb{C}}} \in M_{\text {comp }}$ denote a point represented by $z \in \mathbb{C}_{\Delta}^{d}$. Similarly, $[z, v]_{K_{\mathbb{C}}}$ denotes a point in $L_{\text {comp }}$ represented by $(z, v) \in \mathbb{C}_{\Delta}^{d} \times \mathbb{C}$.

Next, we define a meromorphic section $s_{\text {comp }}^{0}$ of $L_{\text {comp }}$ on $M_{\text {comp }}$ by

$$
s_{\text {comp }}^{0}\left([z]_{K_{\mathbb{C}}}\right)=\left[z, \prod_{j=1}^{d} z_{j}^{\lambda_{j}}\right]_{K_{\mathbb{C}}} \in L_{\mathrm{comp}} \text { for } z \in \mathbb{C}_{\Delta}^{d} .
$$

The section $s_{\text {comp }}^{0}$ is holomorphic and non-zero on $M_{\text {comp }}^{0}=\left(\mathbb{C}^{\times}\right)^{d} / K_{\mathbb{C}}$, where $\left(\mathbb{C}^{\times}\right)^{d}=\left\{z \in \mathbb{C}^{d} \mid z_{i} \neq 0\right.$ for $\left.i=1, \ldots, d\right\} \subset \mathbb{C}_{\Delta}^{d}$. So, it induces a holomorphic trivialization of $L_{\text {comp }}$ on $M_{\text {comp }}^{0}$.

For $m \in \Delta \cap\left(\mathfrak{t}^{n}\right)_{\mathbb{Z}}^{*}$, we define a holomorphic section $\sigma^{m}$ of $L_{\text {comp }}$ by

$$
\sigma^{m}\left([z]_{K_{\mathbb{C}}}\right)=\left[z, \prod_{j=1}^{d} z_{j}^{l_{j}(m)}\right]_{K_{\mathbb{C}}} \in L_{\text {comp }} \text { for } z \in \mathbb{C}_{\Delta}^{d} .
$$


It is well known that $\left\{\sigma^{m} \mid m \in \Delta \cap\left(\mathfrak{t}^{n}\right)_{\mathbb{Z}}^{*}\right\}$ is a basis of the space of holomorphic sections $H^{0}\left(L_{\text {comp }}, \bar{\partial}\right)$.

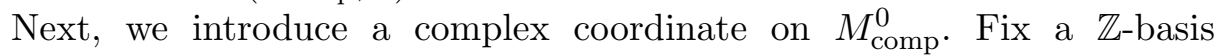
$p_{1}, \ldots, p_{n} \in\left(\mathfrak{t}^{n}\right)_{\mathbb{Z}}^{*}$ and its dual basis $q_{1}, \ldots, q_{n} \in \mathfrak{t}_{\mathbb{Z}}^{n}$ as in Section 5.1. Then, we define a complex coordinate $\varphi_{\text {comp }}^{0}: M_{\text {comp }}^{0} \rightarrow\left(\mathbb{C}^{\times}\right)^{n}$ by

$$
\varphi_{\mathrm{comp}}^{0}\left([z]_{K_{\mathbb{C}}}\right)=\left(\prod_{j=1}^{d} z_{j}^{\left\langle p_{1}, r_{j}\right\rangle}, \ldots, \prod_{j=1}^{d} z_{j}^{\left\langle p_{n}, r_{j}\right\rangle}\right)
$$

where $r_{j} \in \mathfrak{t}_{\mathbb{Z}}^{n}$ is the vector in (5.1) for $j=1, \ldots, d$. Since $\prod_{j=1}^{d} z_{j}^{\left\langle p_{i}, r_{j}\right\rangle}$ is a $K_{\mathbb{C}}$-invariant meromorphic function on $\mathbb{C}^{d}$, it descends to a meromorphic function on $M_{\text {comp }}$. If we set $\left(w_{1}, \ldots, w_{n}\right)=\varphi_{\text {comp }}^{0}\left([z]_{K_{\mathbb{C}}}\right)$, then we have

$$
\sigma^{m}\left([z]_{K_{\mathbb{C}}}\right)=\left(\prod_{i=1}^{n} w_{i}^{\left\langle m, q_{i}\right\rangle}\right) s_{\mathrm{comp}}^{0}\left([z]_{K_{\mathbb{C}}}\right) \text { on } M_{\mathrm{comp}}
$$

5.3. Symplectic potentials. In Sections 5.1 and 5.2 , starting from a Delzant polytope $\Delta$ defined in (5.1), we constructed a symplectic and complex toric manifold, respectively. In this section, we identify them, using symplectic potentials due to $[\mathbf{G u 1 , ~ G u 2 , ~ A b 1 , ~ A b 2 ] . ~ W e ~ a l s o ~ r e c a l l ~ a ~ c e r - ~}$ tain deformation of toric Kähler structures due to [BFMN].

The inclusion $\mu_{K}^{-1}\left(\iota^{*} \lambda_{\Delta}\right) \subset \mathbb{C}_{\Delta}^{d}$ induces a map $\chi_{\text {can }}: M_{\text {symp }} \rightarrow M_{\text {comp. It }}$ is well known that this map is a diffeomorphism. In $[\mathbf{G u 1 , G u 2 ]}$, Guillemin showed that this map is described by a single function $g_{\text {can }}$ as follows.

Fix a $\mathbb{Z}$-basis $p_{1}, \ldots, p_{n} \in\left(\mathfrak{t}^{n}\right)_{\mathbb{Z}}^{*}$ and its dual basis $q_{1}, \ldots, q_{n} \in \mathfrak{t}_{\mathbb{Z}}^{n}$ as in Sections 5.1 and 5.2. Fix $\tilde{q}_{i} \in \mathfrak{t}_{\mathbb{Z}}^{d}$ so that $\pi\left(\tilde{q}_{i}\right)=q_{i}$ for $i=1, \ldots, n$. Let $(x,[\theta])$ be the symplectic coordinate on $M_{\text {symp }}^{0}$ and $\left(w_{1}, \ldots, w_{n}\right)$ the complex coordinate on $M_{\text {comp }}^{0}$ induced by $p_{1}, \ldots, p_{n} \in\left(\mathfrak{t}^{n}\right)_{\mathbb{Z}}^{*}$, respectively. If we write $p=\sum_{i=1}^{n} x_{i} p_{i}$, then, by (5.3) and (5.6) we have

$$
w_{i}\left(\chi_{\operatorname{can}}(x,[\theta])\right)=\prod_{j=1}^{d}\left(\sqrt{\frac{l_{j}(p)}{\pi}} e^{2 \pi \sqrt{-1} \sum_{l=1}^{n}\left\langle u_{j}, \tilde{q}_{l}\right\rangle \theta_{l}}\right)^{\left\langle p_{i}, r_{j}\right\rangle}=e^{2 \pi\left(\frac{\partial g_{\mathrm{can}}}{\partial x_{i}}+\sqrt{-1} \theta_{i}\right)},
$$

where $g_{\text {can }}:$ Int $\Delta \rightarrow \mathbb{R}$ is a function defined by

$$
g_{\text {can }}(p)=\frac{1}{4 \pi} \sum_{j=1}^{d} l_{j}(p) \log l_{j}(p)+\left(\text { a linear function on }\left(\mathfrak{t}^{n}\right)^{*}\right) \text { for } p \in \operatorname{Int} \Delta .
$$

Note that $g_{\text {can }}$ extends continuously to $g_{\text {can }}: \Delta \rightarrow \mathbb{R}$. 
Definition 5.2. A function $g \in C^{0}(\Delta)$ is a symplectic potential if and only if the following holds:

(1) $g-g_{\text {can }} \in C^{\infty}(\Delta)$,

(2) the Hessian $\operatorname{Hess}_{p} g$ of $g$ at $p$ is positive definite for any $p \in \operatorname{Int} \Delta$,

(3) there exists a strictly positive function $\alpha \in C^{\infty}(\Delta)$ such that

$$
\operatorname{det}\left(\operatorname{Hess}_{p} g\right)=\left[\alpha(p) \prod_{j=1}^{d} l_{j}(p)\right]^{-1} \quad \text { for any } p \in \operatorname{Int} \Delta \text {. }
$$

The set of symplectic potentials is denoted by $S P(\Delta)$.

The following results are due to $[\mathbf{G u 1 , ~ G u 2 , ~ A b 1 , ~ A b 2 ] , ~ s u p p l e m e n t e d ~}$ by Baier et al. [BFMN].

Theorem 5.3. Let $\Delta \subset\left(\mathfrak{t}^{n}\right)^{*}$ be a Delzant polytope. Let $\left(M_{\text {symp }}, \omega\right)$ be a symplectic toric manifold and $\left(M_{\mathrm{comp}}, J\right)$ a complex toric manifold constructed from $\Delta$. Let $\left(L_{\mathrm{symp}}, h, \nabla\right)$ be a prequantum line bundle on $M_{\mathrm{symp}}$ and $\left(L_{\mathrm{comp}}, \bar{\partial}\right)$ a holomorphic line bundle on $M_{\text {comp }}$ constructed from $\Delta$. Fix a $\mathbb{Z}$-basis $p_{1}, \ldots, p_{n} \in\left(\mathfrak{t}^{n}\right)_{\mathbb{Z}}^{*}$. Let $(x,[\theta])$ be the symplectic coordinate on $M_{\mathrm{symp}}^{0}$ and $w=\left(w_{1}, \ldots, w_{n}\right)$ the complex coordinate on $M_{\mathrm{comp}}^{0}$ induced by $p_{1}, \ldots, p_{n} \in\left(\mathfrak{t}^{n}\right)_{\mathbb{Z}}^{*}$, respectively.

$(A)$ Each $g \in S P(\Delta)$ defines a $T^{n}$-equivariant diffeomorphism $\chi_{g}: M_{\mathrm{symp}} \rightarrow$ $M_{\text {comp }}$ and a $T^{n}$-equivariant bundle isomorphism $\tilde{\chi}_{g}: L_{\mathrm{symp}} \rightarrow L_{\mathrm{comp}}$ such that the following holds:

(a1) The following diagram commutes:

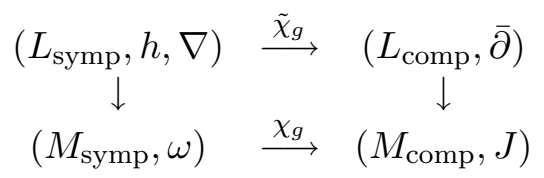

(a2) $\left(M_{\text {symp }}, \omega, \chi_{g}^{*} J\right)$ is a Kähler manifold.

(a3) $\nabla$ is the Chern connection of the Hermitian holomorphic line bundle $\left(L_{\text {symp }}, h, \tilde{\chi}_{g}^{*} \bar{\partial}\right)$.

(a4) $\left.\chi_{g}\right|_{M_{\mathrm{symp}}^{0}}: M_{\mathrm{symp}}^{0} \rightarrow M_{\mathrm{comp}}^{0}$ is a diffeomorphism given by

$$
w_{i}\left(\chi_{g}(x,[\theta])\right)=e^{2 \pi\left(\frac{\partial g}{\partial x_{i}}+\sqrt{-1} \theta_{i}\right)} \text { for } i=1, \ldots, n .
$$

The map $\chi_{g}$ is independent of the choice of the basis $p_{1}, \ldots, p_{n} \in$ $\left(\mathfrak{t}^{n}\right)_{\mathbb{Z}}^{*}$. Moreover, if we write $w_{i}=e^{2 \pi\left(y_{i}+\sqrt{-1} \theta_{i}\right)}$ for $i=1, \ldots, n$, then the inverse mapping $\left(\left.\chi_{g}\right|_{M_{\mathrm{symp}}^{0}}\right)^{-1}: M_{\mathrm{comp}}^{0} \rightarrow M_{\mathrm{symp}}^{0}$ is given by

$$
x_{i}\left(\chi_{g}^{-1}(w)\right)=\frac{\partial f}{\partial y_{i}}, \quad \theta_{i}\left(\left(\chi_{g}^{-1}\right)(w)\right)=\theta_{i} \text { for } i=1, \ldots, n,
$$

where $f(y)=-g(x(y))+\sum_{i=1}^{n} x_{i}(y) y_{i}$. 
(a5) $\tilde{\chi}_{g}^{*} s_{\text {comp }}^{0}=e^{2 \pi\left(g-\sum_{i=1}^{n} x_{i} \frac{\partial g}{\partial x_{i}}\right)} s_{\mathrm{symp}}^{0}$ on $M_{\mathrm{symp}}^{0}$.

(B) On the other hand, if $\chi: M_{\text {symp }} \rightarrow M_{\mathrm{comp}}$ is a $T^{n}$-equivariant diffeomorphism such that $\left(M_{\mathrm{symp}}, \omega, \chi^{*} J\right)$ is a Kähler manifold and that $\chi$ is homotopic to $\chi_{\mathrm{can}}$, then there exists $g \in S P(\Delta)$ such that $\chi=\chi_{g}$.

In [BFMN], the authors considered a certain one-parameter family of symplectic potentials, which provides a one-parameter family of identifications of a symplectic toric manifold with a complex toric manifold. In other words, it provides a deformation of toric Kähler structures. The authors proved the following remarkable property of the deformation.

Proposition 5.4. Let $\chi_{s}: M_{\text {symp }} \rightarrow M_{\text {comp }}$ and $\tilde{\chi}_{s}: L_{\mathrm{symp}} \rightarrow L_{\mathrm{comp}}$ be the diffeomorphism and the bundle isomorphism defined by $g_{s}=g_{0}+s \nu \in$ $S P(\Delta)$ for $s \geq 0$, respectively, where $\nu: \Delta \rightarrow \mathbb{R}$ is a smooth strictly convex function. Then, for each $m \in \Delta \cap\left(\mathfrak{t}^{n}\right)_{\mathbb{Z}}^{*}$, the section $\frac{\tilde{\chi}_{s}^{*} \sigma^{m}}{\left\|\tilde{\chi}_{s}^{*} \sigma^{m}\right\|_{L^{1}\left(M_{\mathrm{symp}}\right)}}$ converges to a delta-function section supported on the fiber $\mu_{T^{n}}^{-1}(m)$ in the following sense: there exists a covariantly constant section $\delta_{m}$ of $\left.\left(L_{\mathrm{symp}}, h, \nabla\right)\right|_{\mu_{T^{n}}^{-1}(m)}$ and a measure $d \theta_{m}$ on $\mu_{T^{n}}^{-1}(m)$ such that, for any smooth section $\phi$ of the dual line bundle $L_{\mathrm{symp}}^{*}$, the following holds:

$$
\lim _{s \rightarrow \infty} \int_{M_{\mathrm{symp}}}\left\langle\phi, \frac{\tilde{\chi}_{s}^{*} \sigma^{m}}{\left\|\tilde{\chi}_{s}^{*} \sigma^{m}\right\|_{L^{1}\left(M_{\mathrm{symp}}\right)}}\right\rangle \frac{\omega^{n}}{n !}=\int_{\mu_{T^{n}(m)}^{-1}}\left\langle\phi, \delta_{m}\right\rangle d \theta_{m} .
$$

Note that the authors proved the above results not only for $m \in \operatorname{Int} \Delta \cap$ $\left(\mathfrak{t}^{n}\right)_{\mathbb{Z}}^{*}$ but also for all $m \in \Delta \cap\left(\mathfrak{t}^{n}\right)_{\mathbb{Z}}^{*}$. In Proposition 6.6 below, we slightly generalize this proposition.

\section{Submanifolds under the deformation due to [BFMN]}

In the last section, starting from a Delzant polytope $\Delta$ defined by (5.1), we constructed a symplectic toric manifold $\left(M_{\text {symp }}, \omega\right)$ and a complex toric manifold $\left(M_{\text {comp }}, J\right)$. In this section, we study the change of the identification $\chi_{s}:\left(M_{\mathrm{symp}}, \omega\right) \rightarrow\left(M_{\mathrm{comp}}, J\right)$ and its lift $\tilde{\chi}_{s}:\left(L_{\mathrm{symp}}, h, \nabla\right) \rightarrow\left(L_{\mathrm{comp}}, \bar{\partial}\right)$ induced by a family of symplectic potentials $g_{s}=g_{0}+s \nu \in S P(\Delta)$ for $s \geq 0$. In Proposition 5.4, $\nu \in C^{\infty}(\Delta)$ is assumed to be a strictly convex function. Here, we assume that $\nu \in C^{\infty}(\Delta)$ is a weakly convex function, which will be important in Propositions 6.5 and 6.6. In particular, we study the behavior of submanifolds and of the prequantum line bundles on them under the change of identification of the ambient toric manifolds.

6.1. Identification of submanifolds. Given a complex submanifold $V_{\text {comp }}$ of $\left(M_{\text {comp }}, J\right)$, we consider the change of the identification $\chi_{s}:\left(M_{\text {symp }}, \omega\right) \rightarrow$ $\left(M_{\text {comp }}, J\right)$. This implies that the complex structure of the complex 
submanifold remains the same, but the symplectic structure $\left(\chi_{s}^{-1}\right)^{*} \omega$ on it changes. In this subsection, we develop a method to identify ( $V_{\text {comp }}$, $\left.\left(\chi_{s}^{-1}\right)^{*} \omega\right)$, for different values of $s$, as symplectic manifolds. We also lift the identification to the prequantum line bundle.

Proposition 6.1. Let $\left(V_{\mathrm{comp}}, J^{V}\right)$ be a compact complex submanifold of $\left(M_{\mathrm{comp}}, J\right)$ and $\rho_{\mathrm{comp}}: V_{\mathrm{comp}} \rightarrow M_{\mathrm{comp}}$ the inclusion. Set $V_{\mathrm{symp}}=$ $\chi_{0}^{-1}\left(V_{\text {comp }}\right)$, and denote the inclusion by $\rho_{0}: V_{\text {symp }} \rightarrow M_{\text {symp }}$.

Then, there exists an inclusion $\rho_{s}: V_{\mathrm{symp}} \rightarrow M_{\mathrm{symp}}$ and a diffeomorphism $\underline{\chi}_{s}: V_{\text {symp }} \rightarrow V_{\text {comp }}$, for each $s \geq 0$, such the following holds:

(a) $\rho_{s}^{*} \omega=\rho_{0}^{*} \omega$.

(b) The following diagram commutes:

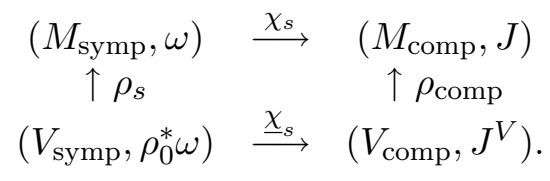

(c) $\left(V_{\text {symp }}, \rho_{0}^{*} \omega, \underline{\chi}_{s}^{*} J^{V}\right)$ is a Kähler manifold.

(d) The maps $\rho_{s}$ and $\underline{\chi}_{s}$ are canonically defined and depend smoothly on $s \geq 0$.

Proof. If we set $\psi_{s}=\chi_{0} \circ\left(\chi_{s}\right)^{-1}: M_{\text {comp }} \rightarrow M_{\text {comp }}$ and $\omega_{s}=\left(\left(\chi_{s}\right)^{-1}\right)^{*} \omega \in$ $\Omega^{2}\left(M_{\text {comp }}\right)$ for each $s \geq 0$, then we have

$$
\psi_{s}^{*} \omega_{0}=\omega_{s}
$$

We show the following.

Claim 6.2. There exists a diffeomorphism $\phi_{s}: V_{\text {comp }} \rightarrow V_{\text {comp for each }}$ $s \geq 0$, such that $\phi_{0}=i d_{V_{\mathrm{comp}}}$ and $\phi_{s}^{*}\left(\rho_{\mathrm{comp}}^{*} \omega_{s}\right)=\rho_{\mathrm{comp}}^{*} \omega_{0}$.

Proof of Claim 6.2. Since $\rho_{\text {comp }}^{*} \omega_{s}$ and $\rho_{\text {comp }}^{*} \omega_{0}$ are cohomologous, Claim 6.2 is a direct consequence of the theorem of Moser. However, we give a proof, because we will use the notation here in the proof of Proposition 6.3.

Define a vector field $X_{s} \in \mathcal{X}\left(M_{\text {comp }}\right)$ by

$$
\left(X_{s}\right)_{\psi_{s}(p)}=\left.\frac{d}{d t}\right|_{t=0} \psi_{s+t}(p) \in T_{\psi_{s}(p)} M_{\text {comp }} \text { for } p \in M_{\text {comp }}
$$

By (6.1) we have

$$
\frac{d \omega_{s}}{d s}=\psi_{s}^{*}\left(L_{X_{s}} \omega_{0}\right)=d \eta_{s}, \text { where } \eta_{s}=\psi_{s}^{*}\left\{i\left(X_{s}\right) \omega_{0}\right\} \in \Omega^{1}\left(M_{\mathrm{comp}}\right) .
$$

Suppose that there exists a diffeomorphism $\phi_{s}: V_{\text {comp }} \rightarrow V_{\text {comp }}$ such that $\phi_{s}^{*}\left(\rho_{\text {comp }}^{*} \omega_{s}\right)=\rho_{\text {comp }}^{*} \omega_{0}$ for each $s \geq 0$. If we define a vector field 
$Y_{s} \in \mathcal{X}\left(V_{\text {comp }}\right)$ by $\left(Y_{s}\right)_{\phi_{s}(p)}=\left.\frac{d}{d t}\right|_{t=0} \phi_{s+t}(p) \in T_{\phi_{s}(p)} V_{\text {comp }}$ for $p \in V_{\text {comp }}$, then we have

$$
\begin{aligned}
0=\frac{d}{d s}\left\{\phi_{s}^{*}\left(\rho_{\mathrm{comp}}^{*} \omega_{s}\right)\right\} & =\phi_{s}^{*}\left\{L_{Y_{s}}\left(\rho_{\mathrm{comp}}^{*} \omega_{s}\right)+\frac{d \rho_{\mathrm{comp}}^{*} \omega_{s}}{d s}\right\} \\
& =\phi_{s}^{*} d\left\{i\left(Y_{s}\right)\left(\rho_{\mathrm{comp}}^{*} \omega_{s}\right)+\rho_{\mathrm{comp}}^{*} \eta_{s}\right\} .
\end{aligned}
$$

Therefore, if we define $Y_{s} \in \mathcal{X}\left(V_{\text {comp }}\right)$ conversely by

$$
i\left(Y_{s}\right)\left(\rho_{\mathrm{comp}}^{*} \omega_{s}\right)+\rho_{\mathrm{comp}}^{*} \eta_{s}=0
$$

then we have a desired diffeomorphism $\phi_{s}: V_{\text {comp }} \rightarrow V_{\text {comp }}$ by integrating $Y_{s} \in \mathcal{X}\left(V_{\text {comp }}\right)$. Moreover, we have $\phi_{0}=i d_{V_{\text {comp }}}$ from this construction.

Since $\left.\rho_{\text {comp }} \circ \chi_{0}\right|_{V_{\text {symp }}}=\chi_{0} \circ \rho_{0}$, we have $\left(\left.\chi_{0}\right|_{V_{\text {symp }}}\right)^{*}\left(\rho_{\text {comp }}^{*} \omega_{0}\right)=\rho_{0}^{*} \omega$. Define a smooth map $\rho_{s}: V_{\text {symp }} \rightarrow M_{\text {symp }}$ by $\rho_{s}=\left.\left(\chi_{s}\right)^{-1} \circ \rho_{\text {comp }} \circ \phi_{s} \circ \chi_{0}\right|_{V_{\text {symp }}}$. By Claim 6.2, we have

$$
\rho_{s}^{*} \omega=\left(\left.\chi_{0}\right|_{V_{\mathrm{symp}}}\right)^{*} \phi_{s}^{*}\left(\rho_{\mathrm{comp}}^{*} \omega_{s}\right)=\left(\left.\chi_{0}\right|_{V_{\mathrm{symp}}}\right)^{*}\left(\rho_{\mathrm{comp}}^{*} \omega_{0}\right)=\rho_{0}^{*} \omega .
$$

Thus, we proved $(a)$.

Define $\underline{\chi}_{s}: V_{\text {symp }} \rightarrow V_{\text {comp }}$ by $\underline{\chi}_{s}=\left.\phi_{s} \circ \chi_{0}\right|_{V_{\text {symp }}}$. Then, we have $\chi_{s} \circ \rho_{s}=$ $\rho_{\text {comp }} \circ \underline{\chi}_{s}$, which implies $(b)$.

Since $\underline{\chi}_{s}^{*}\left(\rho_{\text {comp }}^{*} \omega_{s}\right)=\rho_{s}^{*}\left(\chi_{s}^{*} \omega_{s}\right)=\rho_{s}^{*} \omega=\rho_{0}^{*} \omega$, we see that $\left(V_{\text {symp }}, \rho_{0}^{*} \omega, \underline{\chi}_{s}^{*} J^{V}\right)$ is isomorphic to $\left(V_{\text {comp }}, \rho_{\text {comp }}^{*} \omega_{s}, J^{V}\right)$, which a Kähler manifold. Therefore, (c) follows.

Finally, we prove $(d)$. In the above construction of $\phi_{s}$, there is no ambiguous choice. So, $\phi_{s}$ is canonically defined and depends smoothly on $s \geq 0$. Therefore, the maps $\rho_{s}$ and $\underline{\chi}_{s}$ are canonically defined and depend smoothly on $s \geq 0$. Thus, we finish the proof of Proposition 6.1.

Next, we construct a lift of the maps $\rho_{s}: V_{\text {symp }} \rightarrow M_{\text {symp }}$ and $\underline{\chi}_{s}: V_{\text {symp }} \rightarrow$ $V_{\text {comp }}$ to the prequantum line bundle.

Proposition 6.3. In addition to the assumption of Proposition 6.1, let $\left(L_{\text {symp }}^{V}, h^{V}, \nabla^{V}\right)=\rho_{0}^{*}\left(L_{\text {symp }}, h, \nabla\right)$ and $\left(L_{\text {comp }}^{V}, \bar{\partial}^{V}\right)=\rho_{\text {comp }}^{*}\left(L_{\text {comp }}, \bar{\partial}\right)$ be a prequantum line bundle on $\left(V_{\mathrm{symp}}, \rho_{0}^{*} \omega\right)$ and a holomorphic line bundle on $\left(V_{\text {comp }}, J^{V}\right)$, respectively. Let $\tilde{\rho}_{\text {comp }}: L_{\text {comp }}^{V} \rightarrow L_{\text {comp }}$ be the canonical lift of the inclusion $\rho_{\text {comp }}: V_{\text {comp }} \rightarrow M_{\text {comp }}$.

Then, there exists a lift $\tilde{\rho}_{s}: L_{\text {symp }}^{V} \rightarrow L_{\text {symp }}$ of $\rho_{s}: V_{\text {symp }} \rightarrow M_{\text {symp }}$ and a lift $\underline{\tilde{\chi}}_{s}: L_{\mathrm{symp}}^{V} \rightarrow L_{\mathrm{comp}}^{V}$ of $\underline{\chi}_{s}: V_{\mathrm{symp}} \rightarrow V_{\mathrm{comp}}$, for each $s \geq 0$, such that the following holds:

(a) $\tilde{\rho}_{s}^{*}\left(L_{\text {symp }}, h, \nabla\right)=\left(L_{\text {symp }}^{V}, h^{V}, \nabla^{V}\right)$. 
(b) The following diagram commutes:

$$
\begin{aligned}
& \left(L_{\mathrm{symp}}, h, \nabla\right) \quad \stackrel{\tilde{\chi}_{s}}{\longrightarrow} \quad\left(L_{\mathrm{comp}}, \bar{\partial}\right) \\
& \uparrow \tilde{\rho}_{s} \quad \uparrow \tilde{\rho}_{\mathrm{comp}} \\
& \left(L_{\text {symp }}^{V}, h^{V}, \nabla^{V}\right) \stackrel{\tilde{\tilde{x}}_{s}}{\longrightarrow}\left(L_{\text {comp }}^{V}, \bar{\partial}^{V}\right) .
\end{aligned}
$$

(c) $\nabla^{V}$ is the Chern connection of $\left(L_{\mathrm{symp}}^{V}, h^{V}, \tilde{\tilde{\chi}}_{s}^{*} \bar{\partial}^{V}\right)$.

(d) The maps $\tilde{\rho}_{s}$ and $\underline{\tilde{\chi}}_{s}$ are canonically defined and depend smoothly on $s \geq 0$.

Proof. We use the same notation as in the proof of Proposition 6.1.

First, we show the following.

Claim 6.4. Let $R: V_{\mathrm{symp}} \times[0, \infty) \rightarrow M_{\mathrm{symp}}$ be the map defined by $R(p, s)=$ $\rho_{s}(p)$. Then, the following holds:

$$
i\left(\frac{\partial}{\partial s}\right)\left(R^{*} \omega\right)=0 \text { on } V_{\text {symp }} \times[0, \infty) .
$$

Proof of Claim 6.4. Define $\theta: V_{\text {comp }} \times[0, \infty) \rightarrow M_{\text {comp }}$ by $\theta(p, s)=\psi_{s} \circ$ $\rho_{\text {comp }} \circ \phi_{s}(p)$. Fix any $\left(p_{0}, s_{0}\right) \in V_{\text {symp }} \times[0, \infty)$ and $\underline{v} \in T_{p_{0}} V_{\text {symp }}$. We set $q_{0}=\underline{\chi}_{0}\left(p_{0}\right) \in V_{\text {comp }}, \underline{w}=\left(\underline{\chi}_{0}\right)_{* p_{0}}(\underline{v}) \in T_{q_{0}} V_{\text {comp }}$, and

$$
\begin{gathered}
v=(\underline{v}, 0) \in T_{p_{0}} V_{\text {symp }} \oplus \mathbb{R} \cong T_{\left(p_{0}, s_{0}\right)}\left\{V_{\text {symp }} \times[0, \infty)\right\}, \\
w=(\underline{w}, 0) \in T_{q_{0}} V_{\text {comp }} \oplus \mathbb{R} \cong T_{\left(q_{0}, s_{0}\right)}\left\{V_{\text {comp }} \times[0, \infty)\right\} .
\end{gathered}
$$

Since $R(p, s)=\left(\left(\chi_{0}\right)^{-1} \circ \theta\right)\left(\underline{\chi}_{0}(p), s\right)$, we have

$$
\left\{i\left(\frac{\partial}{\partial s}\right)\left(R^{*} \omega\right)\right\}_{\left(p_{0}, s_{0}\right)}(v)=\omega_{0}\left(\theta_{*\left(q_{0}, s_{0}\right)}\left(\frac{\partial}{\partial s}\right), \theta_{*\left(q_{0}, s_{0}\right)}(w)\right) .
$$

By (6.2), we have

$$
\begin{aligned}
\theta_{*\left(q_{0}, s_{0}\right)}\left(\frac{\partial}{\partial s}\right) & =\left.\frac{\partial}{\partial s}\right|_{s=s_{0}}\left(\psi_{s} \circ \rho_{\text {comp }} \circ \phi_{s}\right)\left(q_{0}\right) \\
& =\left(X_{s_{0}}\right)_{\psi_{s_{0}} \circ \rho_{\mathrm{comp}} \circ \phi_{s_{0}}\left(q_{0}\right)}+\left(\psi_{s_{0}}\right)_{*}\left(\rho_{\mathrm{comp}}\right)_{*}\left(\left.\frac{\partial}{\partial s}\right|_{s=s_{0}} \phi_{s}\left(q_{0}\right)\right), \\
\theta_{*\left(q_{0}, s_{0}\right)}(w) & =\left(\psi_{s_{0}}\right)_{*}\left(\rho_{\mathrm{comp}}\right)_{*}\left(\phi_{s_{0}}\right)_{* q_{0}}(\underline{w}) .
\end{aligned}
$$


Thus, we have

$$
\begin{aligned}
\left\{i\left(\frac{\partial}{\partial s}\right)\left(R^{*} \omega\right)\right\}_{\left(p_{0}, s_{0}\right)}(v) \\
=\left\{\psi_{s_{0}}^{*}\left(i\left(X_{s_{0}}\right) \omega_{0}\right)\right\}_{\rho_{\mathrm{comp}} \circ \phi_{s_{0}}\left(q_{0}\right)}\left(\left(\rho_{\mathrm{comp}}\right)_{*}\left(\phi_{s_{0}}\right)_{* q_{0}}(\underline{w})\right) \\
\quad+\left\{\left(\rho_{\mathrm{comp}}\right)^{*}\left(\psi_{s_{0}}\right)^{*} \omega_{0}\right\}\left(\left(Y_{s_{0}}\right)_{\phi_{s_{0}}\left(q_{0}\right)},\left(\phi_{s_{0}}\right)_{* q_{0}}(\underline{w})\right) \\
=\left(\eta_{s_{0}}\right)_{\rho_{\mathrm{comp}} \circ \phi_{s_{0}}\left(q_{0}\right)}\left(\left(\rho_{\mathrm{comp}}\right)_{*}\left(\phi_{s_{0}}\right)_{* q_{0}}(\underline{w})\right) \\
\quad+\left(-\rho_{\mathrm{comp}}^{*} \eta_{s_{0}}\right)_{\phi_{s_{0}}\left(q_{0}\right)}\left(\left(\phi_{s_{0}}\right)_{* q_{0}}(\underline{w})\right) \\
=0
\end{aligned}
$$

where we used (6.3) and (6.4) in the second equality. This implies Claim 6.4.

Consider the line bundle $\left(L_{\text {symp }}^{\prime}, h^{\prime}, \nabla^{\prime}\right)=R^{*}\left(L_{\text {symp }}, h, \nabla\right)$ on $V_{\text {symp }} \times$ $[0, \infty)$. Let $S^{\prime} \subset L_{\text {symp }}^{\prime}$ be the unit sphere bundle and $p: S^{\prime} \rightarrow V_{\text {symp }} \times[0, \infty)$ the projection. If we denote the connection form of $\nabla^{\prime}$ by $\alpha \in \Omega^{1}\left(S^{\prime}\right)$, then we have $d \alpha=p^{*} R^{*} \omega$. If we denote the horizontal lift of $\frac{\partial}{\partial s} \in \mathcal{X}\left(V_{\text {symp }} \times[0, \infty)\right)$ by $\xi \in \mathcal{X}\left(S^{\prime}\right)$, we have $i(\xi) \alpha=0$ and $p_{*} \xi=\frac{\partial}{\partial s}$. So, we have

$L_{\xi} \alpha=i(\xi) d \alpha=i(\xi)\left(p^{*} R^{*} \omega\right)=p^{*}\left\{i\left(p_{*} \xi\right)\left(R^{*} \omega\right)\right\}=p^{*}\left\{i\left(\frac{\partial}{\partial s}\right)\left(R^{*} \omega\right)\right\}=0$.

Thus, the flow defined by the vector field $\xi \in \mathcal{X}\left(S^{\prime}\right)$ preserves the connection $\nabla^{\prime}$. So, it induces a lift $\tilde{\rho}_{s}: L_{\text {symp }}^{V} \rightarrow L_{\text {symp }}$ of the map $\rho_{s}: V_{\text {symp }} \rightarrow M_{\text {symp }}$ such that $\tilde{\rho}_{s}^{*}(h, \nabla)=\left(h^{V}, \nabla^{V}\right)$ for $s \geq 0$. This implies $(a)$.

Since $\chi_{s} \circ \rho_{s}=\rho_{\text {comp }} \circ \underline{\chi}_{s}$ holds, $\underline{\tilde{\chi}}_{s}=\tilde{\rho}_{\text {comp }}^{-1} \circ \tilde{\chi}_{s} \circ \tilde{\rho}_{s}: L_{\text {symp }}^{V} \rightarrow L_{\text {comp }}^{V}$ is well defined. Thus, $(b)$ holds from the definition of $\underline{\tilde{\chi}}_{s}: L_{\mathrm{symp}}^{V} \rightarrow L_{\mathrm{comp}}^{V}$.

Since $\underline{\tilde{\chi}}_{s}^{*} \bar{\partial}^{V}=\underline{\tilde{\chi}}_{s}^{*}\left(\tilde{\rho}_{\text {comp }}^{*} \bar{\partial}\right)=\tilde{\rho}_{s}^{*}\left(\tilde{\chi}_{s}^{*} \bar{\partial}\right)$, we see that $\left(L_{\text {symp }}^{V}, h^{V}, \underline{\tilde{\chi}}_{s}^{*} \bar{\partial}^{V}\right)$ is isomorphic to $\tilde{\rho}_{s}^{*}\left(L_{\mathrm{symp}}, h, \tilde{\chi}_{s}^{*} \bar{\partial}\right)$. Since $\nabla$ is the Chern connection of $\left(L_{\text {symp }}, h, \tilde{\chi}_{s}^{*} \bar{\partial}\right), \nabla^{V}=\tilde{\rho}_{s}^{*} \nabla$ is the Chern connection of $\left(L_{\text {symp }}^{V}, h^{V}, \underline{\tilde{\chi}}_{s}^{*} \bar{\partial}^{V}\right)$. This implies $(c)$.

Finally, $(d)$ holds obviously from the definition of the maps $\tilde{\rho}_{s}$ and $\underline{\tilde{\chi}}_{s}$.

6.2. Toric subvarieties. Let $\left(M_{\text {symp }}, \omega\right)$ and $\left(M_{\text {comp }}, J\right)$ be a symplectic and complex toric manifold, respectively, constructed from a Delzant polytope $\Delta$ defined by (5.1). In this subsection, we study a (possibly singular) toric subvariety $V_{\text {comp }}$ of $\left(M_{\text {comp }}, J\right)$ under the deformation of toric Kähler structures of the ambient toric manifold.

Fix a $\mathbb{Z}$-basis $p_{1}, \ldots, p_{n} \in\left(\mathfrak{t}^{n}\right)_{\mathbb{Z}}^{*}$ and its dual basis $q_{1}, \ldots, q_{n} \in \mathfrak{t}_{\mathbb{Z}}^{n}$. This induces symplectic coordinate $(x,[\theta])$ on $M_{\text {symp }}^{0}$ as in Section 5.1 and complex coordinate $w=\left(w_{1}, \ldots, w_{n}\right)$ on $M_{\text {comp }}^{0}$ as in Section 5.2. Note that $M_{\text {comp }}^{0}$ is the $T_{\mathbb{C}}^{n}$-orbit through $e=(1, \ldots, 1) \in M_{\text {comp }}^{0}$. 
Proposition 6.5. Let $T_{\mathbb{C}}^{l}$ be an l-dimensional subtorus of $T_{\mathbb{C}}^{n}$. Let $\iota^{*}$ : $\left(\mathfrak{t}^{n}\right)^{*} \rightarrow\left(\mathfrak{t}^{l}\right)^{*}$ be the dual map of the inclusion of the Lie algebra $\iota: \mathfrak{t}^{l} \rightarrow \mathfrak{t}^{n}$. Let $V_{\text {comp }} \subset M_{\text {comp }}$ be a closed l-dimensional (possibly singular) toric subvariety containing $e=(1, \ldots, 1)$. The torus action on $V_{\text {comp }}$ is the restriction of the $T_{\mathbb{C}}^{l}$-action on $M_{\mathrm{comp}}$ and its orbit through e is open dense in $V_{\text {comp. }}$.

(1) Let $\chi_{s}: M_{\mathrm{symp}} \rightarrow M_{\mathrm{comp}}$ be the diffeomorphism defined by

$$
g_{s}=g_{0}+s\left(\underline{\nu} \circ \iota^{*}\right) \in S P(\Delta) \text { for } s \geq 0,
$$

where $\underline{\nu}: \iota^{*}(\Delta) \rightarrow \mathbb{R}$ is a smooth strictly convex function. Set $V_{\mathrm{symp}}=$ $\left(\chi_{0}\right)^{-1}\left(V_{\text {comp }}\right)$. Then, $\left.\chi_{0} \circ \chi_{s}^{-1}\right|_{V_{\text {comp }}}: V_{\text {comp }} \rightarrow V_{\text {comp }}$ is a homeomorphism for each $s \geq 0$.

(2) Let $\rho_{s}: V_{\text {symp }} \rightarrow M_{\text {symp }}$ and $\chi_{s}: V_{\text {symp }} \rightarrow V_{\text {comp }}$ be the maps constructed in Proposition 6.1. Then, $\rho_{s}=\rho_{0}$ and $\underline{\chi}_{s}=\left.\chi_{s}\right|_{V_{\text {symp }}}$ hold for $s \geq 0$. Moreover, their lifts constructed in Proposition 6.3 are given by $\tilde{\rho}_{s}=\tilde{\rho}_{0}: L_{\mathrm{symp}}^{V} \rightarrow L_{\mathrm{symp}}$ and $\underline{\tilde{\chi}}_{s}=\left.\tilde{\chi}_{s}\right|_{L_{\mathrm{symp}}^{V}}: L_{\mathrm{symp}}^{V} \rightarrow L_{\mathrm{comp}}^{V}$.

Proof. (1) Note that $V_{\text {comp }} \cap M_{\text {comp }}^{0}$ is a connected component of

$$
\left\{w \in M_{\text {comp }}^{0} \mid \prod_{i=1}^{n} w_{i}^{\left\langle p, q_{i}\right\rangle}=1 \text { for all } p \in \operatorname{ker} \iota^{*} \cap\left(\mathfrak{t}^{n}\right)_{\mathbb{Z}}^{*}\right\},
$$

which contains $e=(1, \ldots, 1)$. By $(5.8)$, we see that $\left(\chi_{s}\right)^{-1}\left(V_{\text {comp }} \cap M_{\text {comp }}^{0}\right)$ is a connected component of

$$
\left\{(x,[\theta]) \in M_{\text {symp }}^{0} \mid e^{2 \pi \sum_{i=1}^{n}\left\langle p, q_{i}\right\rangle\left(\frac{\partial g_{s}}{\partial x_{i}}+\sqrt{-1} \theta_{i}\right)}=1 \text { for all } p \in \operatorname{ker} \iota^{*} \cap\left(\mathfrak{t}^{n}\right)_{\mathbb{Z}}^{*}\right\} .
$$

On the other hand, we have

$$
\sum_{i=1}^{n}\left\langle p, q_{i}\right\rangle \frac{\partial g_{s}}{\partial x_{i}}=\sum_{i=1}^{n}\left\langle p, q_{i}\right\rangle \frac{\partial g_{0}}{\partial x_{i}}+s \sum_{i=1}^{n}\left\langle p, q_{i}\right\rangle \frac{\partial\left(\underline{\nu} \circ \iota^{*}\right)}{\partial x_{i}}=\sum_{i=1}^{n}\left\langle p, q_{i}\right\rangle \frac{\partial g_{0}}{\partial x_{i}}
$$

because $\sum_{i=1}^{n}\left\langle p, q_{i}\right\rangle \frac{\partial}{\partial x_{i}}$ is a differential in the direction of ker $\iota^{*}$ for all $p \in$ ker $\iota^{*} \cap\left(\mathfrak{t}^{n}\right)_{\mathbb{Z}}^{*}$. Therefore, we have $\left(\chi_{s}\right)^{-1}\left(V_{\text {comp }}\right) \subset\left(\chi_{0}\right)^{-1}\left(V_{\text {comp }}\right)=V_{\text {symp }}$. So, we have an injective continuous map $\left.\chi_{0} \circ \chi_{s}^{-1}\right|_{V_{\text {comp }}}: V_{\text {comp }} \rightarrow V_{\text {comp }}$.

Next, we show that the map $\left.\chi_{0} \circ \chi_{s}^{-1}\right|_{V_{\text {comp }}}: V_{\text {comp }} \rightarrow V_{\text {comp }}$ is surjective. Let $V_{\text {comp }}^{0}$ be the $T_{\mathbb{C}^{-}}^{l}$ orbit through $e$. Note that $\left.\chi_{0} \circ \chi_{s}^{-1}\right|_{V_{\text {comp }}}$ is $T^{l}$-equivariant and injective. If we consider the isotropy subgroup at each point, we have $\chi_{0} \circ \chi_{s}^{-1}\left(V_{\text {comp }}^{0}\right) \subset V_{\text {comp }}^{0}$ and $\chi_{0} \circ \chi_{s}^{-1}\left(V_{\text {comp }} \backslash V_{\text {comp }}^{0}\right) \subset$ $V_{\text {comp }} \backslash V_{\text {comp }}^{0}$. Since $\left.\chi_{0} \circ \chi_{s}^{-1}\right|_{V_{\text {comp }}^{0}}$ is a $C^{\infty}$-map and its differential is an isomorphism at each point, $\chi_{0} \circ \chi_{s}^{-1}\left(V_{\text {comp }}^{0}\right)$ is open in $V_{\text {comp }}^{0}$. On the 
other hand, since $V_{\text {comp }}$ is compact, $\chi_{0} \circ \chi_{s}^{-1}\left(V_{\text {comp }}\right)$ is compact. Therefore, we see that $\left.\chi_{0} \circ \chi_{s}^{-1}\right|_{V_{\text {comp }}^{0}}: V_{\text {comp }}^{0} \rightarrow V_{\text {comp }}^{0}$ is surjective. So, we see that $\left.\chi_{0} \circ \chi_{s}^{-1}\right|_{V_{\text {comp }}}: V_{\text {comp }} \rightarrow V_{\text {comp }}$ is surjective.

Since $\left.\chi_{0} \circ \chi_{s}^{-1}\right|_{V_{\text {comp }}}: V_{\text {comp }} \rightarrow V_{\text {comp }}$ is a bijective continuous map and $V_{\text {comp }}$ is a compact Hausdorff space, it is a homeomorphism.

(2) In the proof of Proposition 6.1, we constructed $\phi_{s}: V_{\text {comp }} \rightarrow V_{\text {comp }}$ by integrating the time-dependent vector field $Y_{s} \in \mathcal{X}\left(V_{\text {comp }}\right)$ defined by

$$
i\left(Y_{s}\right)\left(\rho_{\text {comp }}^{*} \omega_{s}\right)+\rho_{\text {comp }}^{*} \eta_{s}=0, \text { where } \eta_{s}=\psi_{s}^{*}\left\{i\left(X_{s}\right) \omega_{0}\right\} \in \Omega^{1}\left(M_{\text {comp }}\right) .
$$

In our situation, the vector field $Y_{s}$ is defined on $V_{\text {comp }}^{0}$. Since $V_{\text {comp }}^{0}$ is non-compact, it is not obvious that $Y_{s}$ is integrated to define the map $\left.\phi_{s}\right|_{V_{\text {comp }}^{0}}: V_{\text {comp }}^{0} \rightarrow V_{\text {comp }}^{0}$. However, we show that this holds in our case and that $\left.\phi_{s}\right|_{V_{\text {comp }}^{0}}$ extends to a homeomorphism $\phi_{s}: V_{\text {comp }} \rightarrow V_{\text {comp }}$.

In the proof of Proposition $6.5(1)$, we showed that $\left.\psi_{s}\right|_{V_{\text {comp }}^{0}}=\chi_{0} \circ$ $\left.\chi_{s}^{-1}\right|_{V_{\text {comp }}^{0}}: V_{\text {comp }}^{0} \rightarrow V_{\text {comp }}^{0}$ is a diffeomorphism. Moreover, by $(6.2)$ the restriction $\left.X_{s}\right|_{V_{\text {comp }}^{0}}$ takes its values in the tangent bundle of $V_{\text {comp }}^{0}$. If we note $(6.1)$, we have

$$
\begin{aligned}
\rho_{\mathrm{comp}}^{*} \eta_{s} & =\rho_{\mathrm{comp}}^{*} \psi_{s}^{*}\left\{i\left(X_{s}\right) \omega_{0}\right\} \\
& =\rho_{\mathrm{comp}}^{*}\left\{i\left(\left(\psi_{s}^{-1}\right)_{*} X_{s}\right) \psi_{s}^{*} \omega_{0}\right\}=i\left(\left(\psi_{s}^{-1}\right)_{*}\left(\left.X_{s}\right|_{V_{\mathrm{comp}}^{0}}\right)\right) \rho_{\mathrm{comp}}^{*} \omega_{s} .
\end{aligned}
$$

Thus, we have

$$
0=i\left(Y_{s}\right)\left(\rho_{\mathrm{comp}}^{*} \omega_{s}\right)+\rho_{\mathrm{comp}}^{*} \eta_{s}=i\left(Y_{s}+\left(\psi_{s}^{-1}\right)_{*}\left(\left.X_{s}\right|_{V_{\mathrm{comp}}^{0}}\right)\right) \rho_{\mathrm{comp}}^{*} \omega_{s}
$$

So, we have

$$
Y_{s}+\left(\psi_{s}^{-1}\right)_{*}\left(\left.X_{s}\right|_{V_{\mathrm{comp}}^{0}}\right)=0 \in \mathcal{X}\left(V_{\mathrm{comp}}^{0}\right)
$$

For any $p \in V_{\text {comp }}^{0}$, we have

$$
\left(Y_{s}\right)_{p}=-\left\{\left(\psi_{s}^{-1}\right)_{*}\left(\left.X_{s}\right|_{V_{\mathrm{comp}}^{0}}\right)\right\}_{p}=-\left.\frac{d}{d t}\right|_{t=0} \psi_{s}^{-1} \circ \psi_{s+t}(p)=\left.\frac{d}{d t}\right|_{t=0} \psi_{s+t}^{-1} \circ \psi_{s}(p) .
$$

Namely, we have $\left(Y_{s}\right)_{\psi_{s}^{-1}(p)}=\left.\frac{d}{d t}\right|_{t=0} \psi_{s+t}^{-1}(p)$. Thus, the vector field $Y_{s}$ on $V_{\text {comp }}^{0}$ is integrated to define $\left.\phi_{s}\right|_{V_{\text {comp }}^{0}}=\left.\psi_{s}^{-1}\right|_{V_{\text {comp }}^{0}}=\left.\chi_{s} \circ \chi_{0}^{-1}\right|_{V_{\text {comp }}^{0}}$. So, $\left.\phi_{s}\right|_{V_{\text {comp }}^{0}}$ is extended to a homeomorphism $\phi_{s}=\left.\chi_{s} \circ \chi_{0}^{-1}\right|_{V_{\text {comp }}}: V_{\text {comp }} \rightarrow$ $V_{\text {comp. }}$. So, we have $\underline{\chi}_{s}=\left.\phi_{s} \circ \chi_{0}\right|_{V_{\text {symp }}}=\left.\chi_{s}\right|_{V_{\text {symp }}}$. Then, the rest of the statement is obvious.

Recall that we defined a holomorphic section $\sigma^{m}$ of $L_{\text {comp }}$ for $m \in \Delta \cap\left(\mathfrak{t}^{n}\right)_{\mathbb{Z}}^{*}$ by (5.5). Then, $\tilde{\chi}_{s}^{*} \sigma^{m}$ is a section of $L_{\text {symp }}$. By Proposition $6.5(2)$, the section $\tilde{\rho}_{s}^{*}\left(\tilde{\chi}_{s}^{*} \sigma^{m}\right)=\tilde{\rho}_{0}^{*}\left(\tilde{\chi}_{s}^{*} \sigma^{m}\right)$ of $L_{\text {symp }}^{V}$ can be written as $\left.\tilde{\chi}_{s}^{*} \sigma^{m}\right|_{V_{\text {symp }}}$. 
Proposition 6.6. In addition to the assumptions in Proposition 6.5, suppose that $\iota^{*}\left(\left(\mathfrak{t}^{n}\right)_{\mathbb{Z}}^{*}\right)=\left(\mathfrak{t}^{l}\right)_{\mathbb{Z}}^{*}$. Set $\mu_{T^{l}}=\iota^{*} \circ \mu_{T^{n}}: M_{\text {symp }} \rightarrow\left(\mathfrak{t}^{l}\right)^{*}$ and $\Delta_{V}=\mu_{T^{l}}\left(V_{\mathrm{symp}}\right)$.

(1) For $m, m^{\prime} \in \Delta \cap\left(\mathfrak{t}^{n}\right)_{\mathbb{Z}}^{*},\left.\sigma^{m}\right|_{V_{\text {comp }}}=\left.\sigma^{m^{\prime}}\right|_{V_{\text {comp }}}$ if $\iota^{*} m=\iota^{*} m^{\prime}$.

(2) For $p \in \operatorname{Int} \Delta_{V}, \mu_{T^{l}}^{-1}(p) \cap V_{\text {symp }}$ is a Bohr-Sommerfeld fiber for the prequantum line bundle $\left.\left(L_{\mathrm{symp}}, h, \nabla\right)\right|_{V_{\mathrm{symp}}}$ if and only if $p \in \operatorname{Int} \Delta_{V} \cap\left(\mathfrak{t}^{l}\right)_{\mathbb{Z}}^{*}$.

3) Fix any $m \in \Delta \cap\left(\mathfrak{t}^{n}\right)_{\mathbb{Z}}^{*}$ with $\iota^{*} m \in \operatorname{Int} \Delta_{V} \cap\left(\mathfrak{t}^{l}\right)_{\mathbb{Z}}^{*}$. Let $B_{\iota^{*} m}$ be an open neighborhood of $\iota^{*} m$ in $\left(\mathfrak{t}^{l}\right)^{*}$. Then, there exists $C_{0}(s)>0$, depending continuously on $s \geq 0$, such that $\lim _{s \rightarrow \infty} C_{0}(s)=0$ and, for arbitrary $s \geq 0$

$$
\left\|\tau_{s}^{m}\right\|_{C^{0}\left(M_{\mathrm{symp}} \backslash \mu_{T^{l}}^{-1}\left(B_{\iota^{*} m}\right)\right)} \leq C_{0}(s),
$$

where $\tau_{s}^{m}=\frac{\tilde{\chi}_{s}^{*} \sigma^{m}}{\left\|\tilde{\chi}_{s}^{*} \sigma^{m} \mid V_{\text {symp }}\right\|_{L^{1}\left(V_{\text {symp }}\right)}}$.

(4) Fix $m \in \Delta \cap\left(\mathfrak{t}^{n}\right)_{\mathbb{Z}}^{*}$ with $\iota^{*} m \in \operatorname{Int} \Delta_{V} \cap\left(\mathfrak{t}^{l}\right)_{\mathbb{Z}}^{*}$. The section $\left.\tau_{s}^{m}\right|_{V_{\text {symp }}}$ converges to a delta-function section supported on the Bohr-Sommerfeld fiber $\mu_{T^{l}}^{-1}\left(\iota^{*} m\right) \cap V_{\mathrm{symp}}$ in the following sense: there exists a covariantly constant section $\delta_{\iota^{*} m}$ of $\left.\left(L_{\mathrm{symp}}, h, \nabla\right)\right|_{\mu_{T^{l}}^{-1}\left(\iota^{*} m\right)}$ and a measure $d \theta_{\iota^{*} m}$ on $\mu_{T^{l}}^{-1}\left(\iota^{*} m\right) \cap V_{\mathrm{symp}}$ such that, for any smooth section $\phi$ of the dual line bundle $\left(L_{\mathrm{symp}}^{V}\right)^{*}$, the following holds:

$$
\lim _{s \rightarrow \infty} \int_{V_{\mathrm{symp}}}\left\langle\phi,\left.\tau_{s}^{m}\right|_{V_{\mathrm{symp}}}\right\rangle \frac{\left(\rho_{0}^{*} \omega\right)^{l}}{l !}=\int_{\mu_{T^{l}}^{-1}\left(\iota^{*} m\right) \cap V_{\mathrm{symp}}}\left\langle\phi, \delta_{\iota^{*} m}\right\rangle d \theta_{\iota^{*} m} .
$$

Proof. (1) By (5.7), we have $\sigma^{m^{\prime}} / \sigma^{m}=\prod_{i=1}^{n} w_{i}^{\left\langle m^{\prime}-m, q_{i}\right\rangle}$ on $M_{\text {comp }}^{0}$. Since $m^{\prime}-m \in \operatorname{ker} \iota^{*}$, due to (6.5), we have $\sigma^{m^{\prime}} / \sigma^{m}=1$ on $V_{\text {comp }}$.

(2) Since $\iota^{*}\left(\left(\mathfrak{t}^{n}\right)_{\mathbb{Z}}^{*}\right)=\left(\mathfrak{t}^{l}\right)_{\mathbb{Z}}^{*}$, we can take $p_{1}^{\prime}, \ldots, p_{l}^{\prime} \in\left(\mathfrak{t}^{n}\right)_{\mathbb{Z}}^{*}$ so that $\iota^{*} p_{1}^{\prime}, \ldots, \iota^{*} p_{l}^{\prime}$ is a $\mathbb{Z}$-basis of $\left(\mathfrak{t}^{l}\right)_{\mathbb{Z}}^{*}$. In addition, if we fix a $\mathbb{Z}$-basis $p_{l+1}^{\prime}, \ldots, p_{n}^{\prime}$ of $\left(\operatorname{ker} \iota^{*}\right) \cap$ $\left(\mathfrak{t}^{n}\right)_{\mathbb{Z}}^{*}$, then $p_{1}^{\prime}, \ldots, p_{n}^{\prime}$ is a $\mathbb{Z}$-basis of $\left(\mathfrak{t}^{n}\right)_{\mathbb{Z}}^{*}$. It induces the complex coordinate $w^{\prime}=\left(w_{1}^{\prime}, \ldots, w_{n}^{\prime}\right)$ on $M_{\text {comp }}^{0}$ and the symplectic coordinate $\left(x^{\prime},\left[\theta^{\prime}\right]\right)$ on $M_{\text {symp }}^{0}$ as in the previous subsections.

By (6.5), we have $V_{\text {comp }} \cap M_{\text {comp }}^{0}=\left\{w^{\prime} \in M_{\text {comp }}^{0} \mid w_{l+1}^{\prime}=\cdots=w_{n}^{\prime}=\right.$ $1\}$. So, by (6.6), we see that $\theta_{l+1}^{\prime}, \ldots, \theta_{n}^{\prime}$ are constant on $V_{\text {symp }} \cap M_{\text {symp }}^{0}$. Moreover, by (5.4), we have $\mu_{T^{l}}\left(x^{\prime},\left[\theta^{\prime}\right]\right)=\sum_{i=1}^{l} x_{i}^{\prime} p_{i}^{\prime}$ for $\left(x^{\prime},\left[\theta^{\prime}\right]\right) \in M_{\text {symp }}^{0}$. For each $p=\sum_{i=1}^{l} x_{i}^{\prime} p_{i}^{\prime} \in \operatorname{Int} \Delta_{V}$, since $\mu_{T^{l}}^{-1}(p) \cap V_{\text {symp }}$ is a single $T^{l}$-orbit, $x_{1}^{\prime}, \ldots, x_{n}^{\prime}$ are also constant on $\mu_{T^{l}}^{-1}(p) \cap V_{\text {symp }}$. 
On the other hand, due to Lemma 5.1 , we see that $\left.\nabla\right|_{M_{\mathrm{symp}}^{0}}=d-$ $2 \pi \sqrt{-1} \sum_{i=1}^{n} x_{i}^{\prime} d \theta_{i}^{\prime}$ with respect to the trivialization defined by $s_{\text {symp }}^{0}$. Therefore, for a fixed $p=\sum_{i=1}^{l} x_{i}^{\prime} \iota^{*} p_{i}^{\prime} \in \operatorname{Int} \Delta_{V}$, the multi-valued section $\delta_{p}\left(\left[\theta^{\prime}\right]\right)=e^{2 \pi \sqrt{-1} \sum_{i=1}^{l} x_{i}^{\prime} \theta_{i}^{\prime}} s_{\mathrm{symp}}^{0}$ of $\left.\left(L_{\mathrm{symp}}, h, \nabla\right)\right|_{\mu_{T^{l}}^{-1}(p) \cap V_{\mathrm{symp}}}$ is covariantly constant. Since $\delta_{p}$ is single-valued if and only if $p \in \operatorname{Int} \Delta_{V} \cap\left(\mathfrak{t}^{l}\right)_{\mathbb{Z}}^{*}$, we finish the proof.

(3) The following proof is a slight modification of the argument in [BFMN]. If we write $m=\sum_{i=1}^{n} m_{i}^{\prime} p_{i}^{\prime} \in\left(\mathfrak{t}^{n}\right)_{\mathbb{Z}}^{*}$, due to (5.7) and Theorem 5.3, we have

$$
\begin{aligned}
{\tilde{\chi_{s}}}^{*} \sigma^{m} & =\tilde{\chi}_{s}^{*}\left\{\left(\prod_{i=1}^{n}\left(w_{i}^{\prime}\right)^{m_{i}^{\prime}}\right) s_{\mathrm{comp}}^{0}\right\} \\
& =\left\{\prod_{i=1}^{n} e^{2 \pi m_{i}^{\prime}\left(\frac{\partial g_{s}}{\partial x_{i}^{\prime}}+\sqrt{-1} \theta_{i}^{\prime}\right)}\right\} e^{2 \pi\left(g_{s}-\sum_{i=1}^{n} x_{i}^{\prime} \frac{\partial g_{s}}{\partial x_{i}^{\prime}}\right)} s_{\mathrm{symp}}^{0} \\
& =e^{2 \pi\left(g_{s}-\sum_{i=1}^{n}\left(x_{i}^{\prime}-m_{i}^{\prime}\right) \frac{\partial g_{s}}{\partial x_{i}^{\prime}}\right)} e^{2 \pi \sqrt{-1}\left(\sum_{i=1}^{n} m_{i}^{\prime} \theta_{i}^{\prime}\right)} s_{\mathrm{symp}}^{0} \\
& =e^{-2 \pi s \alpha_{m}\left(x^{\prime}\right) \varsigma^{m}}
\end{aligned}
$$

where

$$
\begin{aligned}
& \varsigma^{m}\left(x^{\prime},\left[\theta^{\prime}\right]\right)=e^{2 \pi\left(g_{0}-\sum_{i=1}^{n}\left(x_{i}^{\prime}-m_{i}^{\prime}\right) \frac{\partial g_{0}}{\partial x_{i}^{\prime}}\right)} e^{2 \pi \sqrt{-1}\left(\sum_{i=1}^{n} m_{i}^{\prime} \theta_{i}^{\prime}\right)} s_{\mathrm{symp}}^{0}\left(x^{\prime},\left[\theta^{\prime}\right]\right), \\
& \alpha_{m}\left(x^{\prime}\right)=\sum_{i=1}^{n}\left(x_{i}^{\prime}-m_{i}^{\prime}\right) \frac{\partial\left(\underline{\nu} \circ \iota^{*}\right)}{\partial x_{i}^{\prime}}\left(x^{\prime}\right)-\left(\underline{\nu} \circ \iota^{*}\right)\left(x^{\prime}\right) .
\end{aligned}
$$

If we set $\underline{\alpha}_{\iota^{*} m}(p)=\sum_{i=1}^{l}\left(x_{i}^{\prime}-m_{i}^{\prime}\right) \frac{\partial \nu}{\partial x_{i}^{\prime}}(p)-\underline{\nu}(p)$ for $p=\sum_{i=1}^{l} x_{i}^{\prime} \iota^{*} p_{i}^{\prime} \in \iota^{*} \Delta \subset$ $\left(\mathfrak{t}^{l}\right)^{*}$, then we have $\alpha_{m}\left(x^{\prime}\right)=\underline{\alpha}_{l^{*} m} \circ \mu_{T^{l}}\left(x^{\prime},\left[\theta^{\prime}\right]\right)$. As in the argument in Section 4 in [BFMN], we have

$$
\begin{aligned}
\underline{\alpha}_{\iota^{*} m}(p) & =\underline{\alpha}_{\iota^{*} m}\left(\iota^{*} m\right)+\int_{0}^{1} \frac{d}{d t} \underline{\alpha}_{\iota^{*} m}\left(\iota^{*} m+t\left(p-\iota^{*} m\right)\right) d t \\
& =-\underline{\nu}\left(\iota^{*} m\right)+\int_{0}^{1} t\left(p-\iota^{*} m\right)\left(\operatorname{Hess}_{\iota^{*} m+t\left(p-\iota^{*} m\right)} \underline{\nu}\right)\left(p-\iota^{*} m\right) d t .
\end{aligned}
$$

Since $\underline{\nu}: \iota^{*}(\Delta) \rightarrow \mathbb{R}$ is strictly convex and $\iota^{*} \Delta$ is compact, if we put $\|p\|^{2}=$ $\sum_{i=1}^{l}\left(x_{i}^{\prime}\right)^{2}$ for $p=\sum_{i=1}^{l} x_{i}^{\prime} p_{i}^{\prime}=\iota^{*} x^{\prime} \in\left(\mathfrak{t}^{l}\right)^{*}$, there exists $C_{1}, C_{2}>0$ such that $-\underline{\nu}\left(\iota^{*} m\right)+C_{1}\left\|p-\iota^{*} m\right\|^{2} \leq \underline{\alpha}_{\iota^{*} m}(p) \leq-\underline{\nu}\left(\iota^{*} m\right)+C_{2}\left\|p-\iota^{*} m\right\|^{2}$ for $p \in \iota^{*} \Delta$.

So, we have

$$
e^{-s \underline{\underline{\alpha}}_{\iota} \iota^{*}(p)} \leq e^{s \underline{\underline{\nu}}\left(\iota^{*} m\right)-s C_{1}\left\|p-\iota^{*} m\right\|^{2}} \text { for } p \in \iota^{*} \Delta .
$$


On the other hand, there exists $C_{3}>0$, for sufficiently small $r>0$

$$
\begin{aligned}
\int_{\Delta_{V}} & e^{-s \underline{\alpha}_{\iota^{*} m}(p)} d p \\
& \geq \int_{B_{r}\left(\iota^{*} m\right) \cap \Delta_{V}} e^{s \underline{\nu}\left(\iota^{*} m\right)-s C_{2}\left\|p-\iota^{*} m\right\|^{2}} d p \geq C_{3} r^{l} e^{s \underline{\nu}\left(\iota^{*} m\right)-s C_{2} r^{2}} .
\end{aligned}
$$

Since $\alpha_{m}=\underline{\alpha}_{\iota^{*} m} \circ \mu_{T^{l}}$ is a smooth function on $M_{\text {symp }}, \varsigma^{m}$ is a smooth section

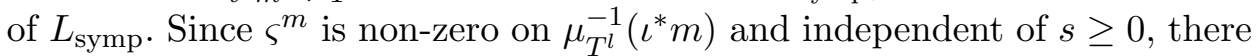
exists $C_{4}>0$ such that

$$
\left\|\left.\tilde{\chi}_{s}^{*} \sigma^{m}\right|_{V_{\mathrm{symp}}}\right\|_{L^{1}\left(V_{\mathrm{symp}}\right)} \geq C_{4} r^{l} e^{s \underline{\underline{\nu}\left(\iota^{*} m\right)-s C_{2} r^{2}} .}
$$

By (6.7) and (6.8), there exists $C_{5}>0$ such that

$$
\begin{aligned}
& \left|\tau_{s}^{m}\left(x^{\prime},\left[\theta^{\prime}\right]\right)\right|=\left|\frac{\tilde{\chi}_{s}^{*} \sigma^{m}\left(x^{\prime},\left[\theta^{\prime}\right]\right)}{\left\|\left.\tilde{\chi}_{s}^{*} \sigma^{m}\right|_{V_{\mathrm{symp}}}\right\|_{L^{1}\left(V_{\mathrm{symp}}\right)}}\right| \\
& \leq C_{5} \frac{e^{s \underline{\nu}\left(\iota^{*} m\right)-s C_{1}\left\|\iota^{*} x^{\prime}-\iota^{*} m\right\|^{2}}}{r^{l} e^{s \underline{\nu}\left(\iota^{*} m\right)-s C_{2} r^{2}}}=C_{5} r^{-l} e^{-s\left(C_{1}\left\|\iota^{*} x^{\prime}-\iota^{*} m\right\|^{2}-C_{2} r^{2}\right)} \text {. }
\end{aligned}
$$

Since we can take small $r>0$ so that $C_{1}\left\|p-\iota^{*} m\right\|^{2}-C_{2} r^{2}>0$ for any $p \in \iota^{*} \Delta \backslash B_{\iota^{*} m}$, we finish the proof.

(4) By the above argument, we also have

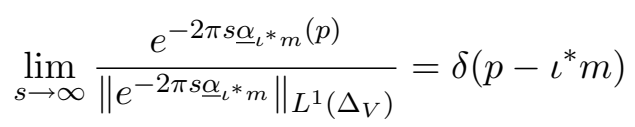

for $\iota^{*} m \in \operatorname{Int} \Delta_{V} \cap\left(\mathfrak{t}^{l}\right)_{\mathbb{Z}}^{*}$, where $\delta(x)$ is the Dirac delta function on $\left(\mathfrak{t}^{l}\right)^{*}$ supported at the origin. Moreover, the restriction $\left.\varsigma^{m}\right|_{\mu_{T^{l}}^{-1}\left(\iota^{*} m\right) \cap V_{\mathrm{symp}}}=$ $\left.c\left(e^{2 \pi \sqrt{-1}\left(\sum_{i=1}^{l} m_{i}^{\prime} \theta_{i}^{\prime}\right)} s_{\text {symp }}^{0}\right)\right|_{\mu_{T^{l}}^{-1}\left(\iota^{*} m\right) \cap V_{\text {symp }}}$, where $c$ is a constant, is a covariantly constant section on $\mu_{T^{l}}^{-1}\left(\iota^{*} m\right) \cap V_{\text {symp }}$, which we denote by $\delta_{\iota^{*} m}\left(\theta^{\prime}\right)$. So, the assertion is proved easily. The details are the same as in [BFMN].

\section{Proof of main result}

In this section, we prove Theorem 2.1 by applying the method developed in Section 6. In Section 7.1, we explain how the setting of Theorem 2.1 fits into the framework of Section 6. In Section 7.2, we construct a family of complex structures on the flag manifold, from which (1)-(3) of Theorem 2.1 turn out to be obvious. Finally, we prove Theorem 2.1 (4) in Sections 7.3 and 7.4.

7.1. Set up. In Section 2, we fixed a symplectic structure $\omega_{\mathbb{P}}$ on $\mathbb{P}=$ $\prod_{l=1}^{n-1} \mathbb{P}\left(\bigwedge^{l} \mathbb{C}^{n}\right)$. We denote the complex structure on $\mathbb{P}$ by $J_{\mathbb{P}}$. Note that 
$\left(\mathbb{P}, \omega_{\mathbb{P}}, J_{\mathbb{P}}\right)$ is a toric Kähler manifold, constructed from a Delzant polytope $\Delta_{\mathbb{P}}$. Moreover, the toric Kähler manifold $\left(\mathbb{P}, \omega_{\mathbb{P}}, J_{\mathbb{P}}\right)$ can be viewed as the identification of a symplectic toric manifold $\left(\mathbb{P}_{\text {symp }}, \omega_{\mathbb{P}}\right)$ with a complex toric manifold $\left(\mathbb{P}_{\text {comp }}, J_{\mathbb{P}}\right)$ by the diffeomorphism $\chi_{0}:\left(\mathbb{P}_{\text {symp }}, \omega_{\mathbb{P}}\right) \rightarrow\left(\mathbb{P}_{\text {comp }}, J_{\mathbb{P}}\right)$ defined by a symplectic potential $g_{0} \in S P\left(\Delta_{\mathbb{P}}\right)$, as in Section 5.3. Similarly, the Hermitian line bundle $\left(L^{\mathbb{P}}, h^{\mathbb{P}}, \nabla^{\mathbb{P}}\right)$ can also be viewed as the identification of the prequantum line bundle $\left(L_{\text {symp }}^{\mathbb{P}}, h^{\mathbb{P}}, \nabla^{\mathbb{P}}\right)$ on $\left(\mathbb{P}_{\text {symp }}, \omega_{\mathbb{P}}\right)$ with the holomorphic line bundle $\left(L_{\text {comp }}^{\mathbb{P}}, \bar{\partial}^{\mathbb{P}}\right)$ on $\left(\mathbb{P}_{\text {comp }}, J_{\mathbb{P}}\right)$ via the bundle isomorphism $\tilde{\chi}_{0}$, which is a lift of the map $\chi_{0}: \mathbb{P}_{\text {symp }} \rightarrow \mathbb{P}_{\text {comp }}$.

The flag manifold $\left(\mathbb{F}, \omega_{\mathbb{F}}, J_{\mathbb{F}}\right)$ in Theorem 2.1 can also be viewed as the identification of $\left(\mathbb{F}_{\text {symp }}, \omega_{\mathbb{F}}\right)$ with $\left(\mathbb{F}_{\text {comp }}, J_{\mathbb{F}}\right)$ as follows. Let us denote the Plücker embedding by $\rho_{\text {comp }}:\left(\mathbb{F}_{\text {comp }}, J_{\mathbb{F}}\right) \rightarrow\left(\mathbb{P}_{\text {comp }}, J_{\mathbb{P}}\right)$. We set $\mathbb{F}_{\text {symp }}=$ $\chi_{0}^{-1}\left(\mathbb{F}_{\text {comp }}\right)$ and let $\rho_{\text {symp }}: \mathbb{F}_{\text {symp }} \rightarrow \mathbb{P}_{\text {symp }}$ be the embedding. Note that $\rho_{\text {symp }}^{*} \omega_{\mathbb{P}}=\omega_{\mathbb{F}}$. We also set $\left(L_{\text {symp }}^{\mathbb{F}}, h^{\mathbb{F}}, \nabla^{\mathbb{F}}\right)=\rho_{\text {symp }}^{*}\left(L_{\text {symp }}^{\mathbb{P}}, h^{\mathbb{P}}, \nabla^{\mathbb{P}}\right)$ and $\left(L_{\text {comp }}^{\mathbb{F}}, \bar{\partial}^{\mathbb{F}}\right)=\rho_{\text {comp }}^{*}\left(L_{\text {comp }}^{\mathbb{P}}, \bar{\partial}^{\mathbb{P}}\right)$. Then, we have the following commutative diagrams:

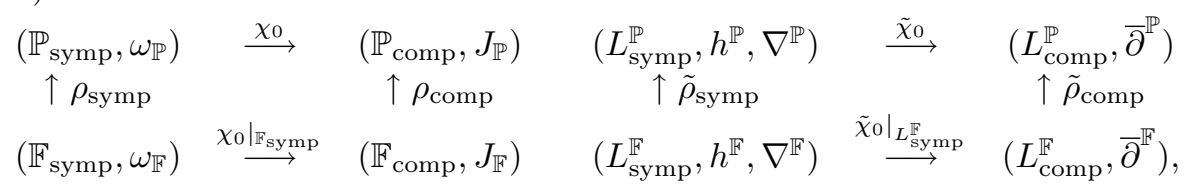

where $\tilde{\rho}_{\text {symp }}$ and $\tilde{\rho}_{\text {comp }}$ are the natural embeddings.

In Section 3.1, we constructed a family of varieties $\left\{F l_{n}(t)=\right.$ $\left.M_{n}(\mathbb{C}) / /{ }_{t} B\right\}_{t \in \mathbb{C}}$. We set $\left(V_{t, \mathrm{comp}}, J_{V_{t}}\right)=F l_{n}(t)$ for $t \in[0,1]$ and denote the deformed Plücker embedding by $\rho_{t, \text { comp }}: V_{t, \text { comp }} \rightarrow \mathbb{P}_{\text {comp }}$, which is defined in Section 3.1. Let $V_{t, \text { symp }}=\chi_{0}^{-1}\left(V_{t, \text { comp }}\right)$ and $\rho_{t, 0}: V_{t, \text { symp }} \rightarrow \mathbb{P}_{\text {symp }}$ be the embedding. We also set $\left(L_{\text {symp }}^{V_{t}}, h^{V_{t}}, \nabla^{V_{t}}\right)=\rho_{t, 0}^{*}\left(L_{\text {symp }}^{\mathbb{P}}, h^{\mathbb{P}}, \nabla^{\mathbb{P}}\right)$ and $\left(L_{\text {comp }}^{V_{t}}, \bar{\partial}^{V_{t}}\right)=\rho_{t, \text { comp }}^{*}\left(L_{\text {comp }}^{\mathbb{P}}, \bar{\partial}^{\mathbb{P}}\right)$. Then, we have a commutative diagram, which is the case $s=0$ in the diagram (7.4) below. Note that $V_{1, \text { comp }}=\mathbb{F}_{\text {comp }}$ and $V_{1, \text { symp }}=\mathbb{F}_{\text {symp }}$ and that $V_{0, \text { comp }}$ is the Gelfand-Cetlin toric variety $F l_{n}(0) \subset \mathbb{P}$. Thus, the above family $\left\{V_{t, \text { comp }}\right\}_{t \in[0,1]}$ connects the flag manifold $\mathbb{F}_{\text {comp }}$ with the Gelfand-Cetlin toric variety $F l_{n}(0)$.

For any $t \in[0,1]$, fix a path $\gamma_{t}:[0,1] \rightarrow \mathbb{C}^{n-1}$, which is given by straight lines connecting the points

$$
\gamma_{t}(0)=(1, \ldots, 1) \rightarrow(1, \ldots, 1, t) \rightarrow(1, \ldots, 1, t, t) \rightarrow \cdots \rightarrow(t, \ldots, t)=\gamma_{t}(1)
$$

Recall that we constructed a family of varieties $\left\{F l_{n}(\tau)=M_{n}(\mathbb{C}) / /{ }_{\tau} B\right\}$ for $\tau \in\left(\mathbb{C}^{\times}\right)^{n-1}$ in Section 3.2 and that we also constructed a degeneration in stages by extending the family. The path $\gamma_{t}$ is an approximation to the path $\gamma_{0}$ for the degeneration in stages. Note that $F l_{n}\left(\gamma_{t}(1)\right)=\left(V_{t, \mathrm{comp}}, J_{V_{t}}\right)$ for 
$t \in[0,1]$. Due to Propositions 4.2 and 4.3 , the gradient-Hamiltonian flow along the path $\gamma_{t}$, for $t \in(0,1]$, gives a symplectic diffeomorphism which together with its lift to the prequantum line bundle gives rise to the following diagram:

$$
\begin{array}{ccc}
\left(L_{\text {symp }}^{\mathbb{F}}, h^{\mathbb{F}}, \nabla^{\mathbb{F}}\right) & \stackrel{\tilde{\Psi}_{t}}{\longrightarrow} & \left(L_{\text {symp }}^{V_{t}}, h^{V_{t}}, \nabla^{V_{t}}\right) \\
\downarrow & & \downarrow \\
\left(\mathbb{F}_{\text {symp }}, \rho_{\text {symp }}^{*} \omega_{\mathbb{P}}\right) & \stackrel{\Psi_{t}}{\longrightarrow} & \left(V_{t, \text { symp }}, \rho_{t, 0}^{*} \omega_{\mathbb{P}}\right) .
\end{array}
$$

We can also extend $\Psi_{t}: \mathbb{F}_{\text {symp }} \rightarrow V_{t, \text { symp }}$ in (7.2) to the case $t=0$ if we restrict its domain to an open dense subset $\mathbb{F}_{\text {symp }}^{\circ}$ of $\mathbb{F}_{\text {symp. }}$. It is already given by (3.2). Using the notation in this section, it should be written as $\Psi_{0}: \mathbb{F}_{\text {symp }}^{\circ} \rightarrow V_{0, \text { symp }}^{\circ}$. We also have its lift to the prequantum line bundle. Thus, we have the following:

$$
\begin{array}{ccc}
\left.\left(L_{\mathrm{symp}}^{\mathbb{F}}, h^{\mathbb{F}}, \nabla^{\mathbb{F}}\right)\right|_{\mathbb{F}_{\text {symp }}^{\circ}} & \stackrel{\tilde{\Psi}_{0}}{\longrightarrow} & \left.\left(L_{\text {symp }}^{V_{0}}, h^{V_{0}}, \nabla^{V_{0}}\right)\right|_{V_{0, \text { symp }}^{\circ}} \\
\downarrow & \downarrow \\
\left(\mathbb{F}_{\text {symp }}^{\circ}, \rho_{\text {symp }}^{*} \omega_{\mathbb{P}}\right) & \stackrel{\Psi_{0}}{\longrightarrow} & \left(V_{0, \text { symp }}^{\circ}, \rho_{0,0}^{*} \omega_{\mathbb{P}}\right) .
\end{array}
$$

7.2. Construction of a family of complex structures. On $\left(\mathbb{P}, \omega_{\mathbb{P}}, J_{\mathbb{P}}\right)$, a $\left(\frac{1}{2} \operatorname{dim}_{\mathbb{R}} \mathbb{P}\right)$-dimensional torus $T_{\mathbb{P}}$ acts with an open dense orbit. On the Gelfand-Cetlin toric variety $F l_{n}(0)=V_{0, \text { comp }} \subset \mathbb{P}$, a $\left(\frac{1}{2} \operatorname{dim}_{\mathbb{R}} \mathbb{F}\right)$-dimensional torus $T_{\mathrm{GC}}$ acts with an open dense subset, as explained in Section 3.2. There is an injective homomorphism $\tilde{\iota}_{\mathrm{GC}}: T_{\mathrm{GC}} \rightarrow T_{\mathbb{P}}$ such that the embedding $\rho_{0, \text { comp }}: V_{0, \text { comp }} \rightarrow \mathbb{P}_{\text {comp }}$ is equivariant. It is described explicitly in Section 6 in [NNU]. Let $\iota_{\mathrm{GC}}^{*}: \mathfrak{t}_{\mathbb{P}}^{*} \rightarrow \mathfrak{t}_{\mathrm{GC}}^{*}$ be the dual map of the inclusion of the Lie algebras $\iota_{\mathrm{GC}}: \mathfrak{t}_{\mathrm{GC}} \rightarrow \mathfrak{t}_{\mathbb{P}}$. From the description of the map $\tilde{\iota}_{\mathrm{GC}}: T_{\mathrm{GC}} \rightarrow T_{\mathbb{P}}$ given in $[\mathbf{N N U}]$, we see that $\iota_{\mathrm{GC}}^{*}\left(\left(\mathfrak{t}_{\mathbb{P}}\right)_{\mathbb{Z}}^{*}\right)=\left(\mathfrak{t}_{\mathrm{GC}}\right)_{\mathbb{Z}}^{*}$.

Fix a strictly convex function $\underline{\nu}: \mathfrak{t}_{\mathrm{GC}}^{*} \rightarrow \mathbb{R}$ and set $\nu=\underline{\nu} \circ \iota_{\mathrm{GC}}^{*}: \mathfrak{t}_{\mathbb{P}}^{*} \rightarrow \mathbb{R}$. Let us consider the diffeomorphism $\chi_{s}:\left(\mathbb{P}_{\text {symp }}, \omega_{\mathbb{P}}\right) \rightarrow\left(\mathbb{P}_{\text {comp }}, J_{\mathbb{P}}\right)$ defined by $g_{s}=g_{0}+s \nu \in S P\left(\Delta_{\mathbb{P}}\right)$. Due to Propositions 6.1 and 6.3 , we have the following commutative diagrams:

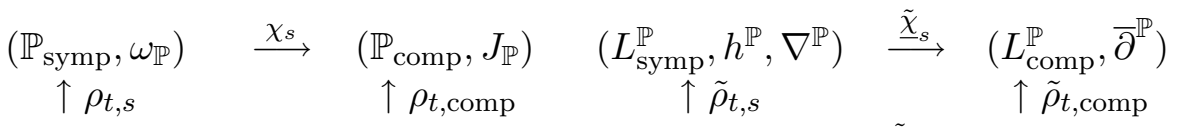

$$
\begin{aligned}
& \left(V_{t, \mathrm{symp}}, \rho_{t, 0}^{*} \omega_{\mathbb{P}}\right) \stackrel{\underline{\chi}_{t, s}}{\longrightarrow}\left(V_{t, \mathrm{comp}}, J_{V_{t}}\right) \quad\left(L_{\mathrm{symp}}^{V_{t}}, h^{V_{t}}, \nabla^{V_{t}}\right) \stackrel{\tilde{\chi}_{t, s}}{\longrightarrow}\left(L_{\text {comp }}^{V_{t}}, \bar{\partial}^{V_{t}}\right),
\end{aligned}
$$

where $\underline{\chi}_{t, 0}=\left.\chi_{0}\right|_{V_{t, \text { symp }}}$ and $\underline{\tilde{\chi}}_{t, 0}=\left.\tilde{\chi}_{0}\right|_{L_{t, \text { symp }}^{V}}$. Note that $\rho_{t, s}^{*} \omega_{\mathbb{P}}=\rho_{t, 0}^{*} \omega_{\mathbb{P}} \in$ $\Omega^{2}\left(V_{t, \text { symp }}\right)$. 
In the case $(t, s)=(1,0)$, the diagrams (7.4) are the same as the diagrams (7.1). In the case $t=0$, the diagrams (7.4) describe the deformation of toric Kähler structures of the Gelfand-Cetlin toric variety $V_{0, \text { comp }}$. The defining equation of the image of the embedding $\rho_{0 \text {,comp }}: V_{0, \text { comp }} \rightarrow \mathbb{P}_{\text {comp }}$ is given by equations (7) in [NNU]. From this description, we see that the image $\rho_{0 \text {,comp }}\left(V_{0, \text { comp }}\right)$ contains the point $(1, \ldots, 1) \in \mathbb{P}$ in the notation in Proposition 6.5. So, Proposition 6.6 can be applied to our case. Therefore, the holomorphic sections on $V_{0, \text { comp }}$ converge to delta-function sections supported on Bohr-Sommerfeld fibers as $s$ goes to infinity. Therefore, the holomorphic sections on $V_{t, \text { comp }}$ are close to delta-function sections when $t$ and $s$ go to zero and infinity, respectively, at the same time. So, we make $t$ a function of $s$ as follows: let $t:[0, \infty) \rightarrow \mathbb{R}_{>0}$ be a strictly decreasing $C^{\infty}$-function with $t(0)=1$ and $\lim _{s \rightarrow \infty} t(s)=0$, where $\mathbb{R}_{>0}$ is the set of positive real numbers. (In fact, $t(s)$ should be required to satisfy additional conditions, which will be discussed in Lemma 7.5 below.)

We define a complex structure $J_{s}$ on $\left(\mathbb{F}_{\text {symp }}, \rho_{\text {symp }}^{*} \omega_{\mathbb{P}}\right)$ as the pull back of $J_{V_{t(s)}}$ by the following composition of diffeomorphisms, which appeared in the diagrams (7.2) and (7.4):

$$
\left(\mathbb{F}_{\text {symp }}, \rho_{\text {symp }}^{*} \omega_{\mathbb{P}}\right) \stackrel{\Psi_{t}}{\longrightarrow}\left(V_{t, \text { symp }}, \rho_{t, 0}^{*} \omega_{\mathbb{P}}\right) \stackrel{\underline{\chi}_{t, s}}{\longrightarrow}\left(V_{t, \text { comp }}, J_{V_{t}}\right) .
$$

Namely, a family of complex structures $\left\{J_{s}\right\}_{s \in[0, \infty)}$ on $\left(\mathbb{F}_{\text {symp }}, \rho_{\text {symp }}^{*} \omega_{\mathbb{P}}\right)$ is defined by

$$
J_{s}=\left(\underline{\chi}_{t(s), s} \circ \Psi_{t(s)}\right)^{*} J_{V_{t(s)}} .
$$

Then, (1) and (2) of Theorem 2.1 follow from the construction of $\left\{J_{s}\right\}_{s \in[0, \infty)}$. By Proposition $6.1(2),\left(V_{t, \text { symp }}, \rho_{t, 0}^{*} \omega_{\mathbb{P}}, \underline{\chi}_{t, s}^{*} J_{V_{t}}\right)$ is a Kähler manifold. Moreover, $\left(V_{t(s), \text { symp }}, \rho_{t(s), 0}^{*} \omega_{\mathbb{P}}, \underline{\chi}_{t(s), s}^{*} J_{V_{t(s)}}\right)$ is isomorphic to $\left(\mathbb{F}_{\mathrm{symp}}, \rho_{\mathrm{symp}}^{*} \omega_{\mathbb{P}}, J_{s}\right)$ as a Kähler manifold. So, Theorem 2.1 (3) follows as well. Thus, for any $s \in[0, \infty), J_{s}$ induces the holomorphic structure $\bar{\partial}^{s}$ of the Hermitian line bundle $\left(L_{\text {symp }}^{\mathbb{F}}, h^{\mathbb{F}}, \nabla^{\mathbb{F}}\right)$. Note that the map $\underline{\tilde{\chi}}_{t(s), s} \circ \tilde{\Psi}_{t(s)}:\left(L_{\text {symp }}^{\mathbb{F}}, \bar{\partial}^{s}\right) \rightarrow$ $\left(L_{\text {comp }}^{V_{t(s)}}, \bar{\partial}^{V_{t(s)}}\right)$ is an isomorphism of holomorphic line bundles.

To prove Theorem 2.1 (4), we have to construct a basis $\left\{\sigma_{s}^{m} \mid m \in \Delta_{\text {GC }} \cap\right.$ $\left.\left(\mathfrak{t}_{\mathrm{GC}}\right)_{\mathbb{Z}}^{*}\right\}$ of the space of holomorphic sections $H^{0}\left(L_{\text {symp }}^{\mathbb{F}}, \bar{\partial}^{s}\right)$.

First, we find a basis of the space of holomorphic sections $H^{0}\left(L_{\text {comp }}^{V_{0}}, \bar{\partial}^{V_{0}}\right)$ in the following way. Recall that the Gelfand-Cetlin polytope $\Delta_{\mathrm{GC}}$ is considered as a subset of $\mathfrak{t}_{\mathrm{GC}}^{*}$ as explained in Section 3.2. Since $\iota_{\mathrm{GC}}^{*}\left(\left(\mathfrak{t}_{\mathbb{P}}\right)_{\mathbb{Z}}^{*}\right)=\left(\mathfrak{t}_{\mathrm{GC}}\right)_{\mathbb{Z}}^{*}$, for each $m \in \Delta_{\mathrm{GC}} \cap\left(\mathfrak{t}_{\mathrm{GC}}\right)_{\mathbb{Z}}^{*}$, we can choose $\tilde{m} \in \Delta_{\mathbb{P}} \cap\left(\mathfrak{t}_{\mathbb{P}}\right)_{\mathbb{Z}}^{*}$ such that $\iota^{*}(\tilde{m})=m$. Let $\sigma^{\tilde{m}}$ be the holomorphic section of $\left(L_{\text {comp }}^{\mathbb{P}}, \bar{\partial}^{\mathbb{P}}\right)$ defined by (5.5). Due to Proposition 6.6 (1), the restriction $\left(\tilde{\rho}_{0, \text { comp }}\right)^{*} \sigma_{\tilde{m}}$ to $V_{0, \text { comp }}$ depends only on $m \in \Delta_{\mathrm{GC}} \cap\left(\mathfrak{t}_{\mathrm{GC}}\right)_{\mathbb{Z}}^{*}$, not on $\tilde{m}$. Due to Corollary 3.3, 
$\Delta_{\mathrm{GC}}$ is the moment polytope for the action of $T_{\mathrm{GC}}$ on the GelfandCetlin toric variety $\left(V_{0, \text { symp }}, \rho_{0,0}^{*} \omega_{\mathbb{P}}\right)$, where $\rho_{0,0}^{*} \omega_{\mathbb{P}}$ is the first Chern form of $\left(L_{\text {symp }}^{V_{0}}, h^{V_{0}}, \nabla^{V_{0}}\right)$. Since $L_{\text {symp }}^{V_{0}}$ is naturally identified with $L_{\text {comp }}^{V_{0}}$ by the map $\underline{\tilde{\chi}}_{0,0}$, we see that $\left\{\left(\tilde{\rho}_{0, \text { comp }}\right)^{*} \sigma_{\tilde{m}} \mid m \in \Delta_{\mathrm{GC}} \cap\left(\mathfrak{t}_{\mathrm{GC}}\right)_{\mathbb{Z}}^{*}\right\}$ is a basis of the space of holomorphic sections $H^{0}\left(L_{\text {comp }}^{V_{0}}, \bar{\partial}^{V_{0}}\right)$ by the general fact on toric varieties.

Since linearly independence of the restriction of holomorphic sections from $\mathbb{P}$ is an open condition, there exists $s_{0}>0$ such that, for any $s \geq s_{0}$, $\left\{\left(\tilde{\rho}_{t(s), \text { comp }}\right)^{*} \sigma^{\tilde{m}} \mid m \in \Delta_{\mathrm{GC}} \cap\left(\mathfrak{t}_{\mathrm{GC}}\right)_{\mathbb{Z}}^{*}\right\}$ are liniarly independent. On the other hand, all $\left(V_{t(s), \text { comp }}, J_{V_{t(s)}}\right)$ and all $\left(L_{\text {comp }}^{V_{t(s)}}, \bar{\partial}^{V_{t(s)}}\right)$ are isomorphic for $s \geq 0$ as complex manifolds and holomorphic line bundles, respectively. Moreover, due to [GS], the dimension of the space of holomorphic sections of $L_{\text {comp }}^{V_{1}}=$ $L_{\text {comp }}^{\mathbb{F}}$ is equal to the numbers of $\Delta_{\mathrm{GC}} \cap\left(\mathfrak{t}_{\mathrm{GC}}\right)_{\mathbb{Z}}^{*}$. Therefore, we conclude that $\left\{\left(\tilde{\rho}_{t(s), \mathrm{comp}}\right)^{*} \sigma^{\tilde{m}} \mid m \in \Delta_{\mathrm{GC}} \cap\left(\mathfrak{t}_{\mathrm{GC}}\right)_{\mathbb{Z}}^{*}\right\}$ is a basis of the space of holomorphic sections $H^{0}\left(L_{\text {comp }}^{V_{t(s)}}, \bar{\partial}^{V_{t(s)}}\right)$ for any $s \geq s_{0}$.

So we define, for $s \geq s_{0}$,

$$
\sigma_{s}^{m}=\left(\underline{\tilde{\chi}}_{t(s), s} \circ \tilde{\Psi}_{t(s)}\right)^{*}\left(\left(\tilde{\rho}_{t(s), \mathrm{comp}}\right)^{*} \sigma^{\tilde{m}}\right) \text { for } m \in \Delta_{\mathrm{GC}} \cap\left(\mathfrak{t}_{\mathrm{GC}}\right)_{\mathbb{Z}}^{*}
$$

Since all $\left(V_{t(s), \text { comp }}, J_{V_{t(s)}}\right)$ and all $\left(L_{\text {comp }}^{V_{t(s)}}, \bar{\partial}^{V_{t(s)}}\right)$ are isomorphic for $s \geq 0$ as complex manifolds and holomorphic line bundles, respectively, we can extend a basis $\left\{\sigma_{s}^{m} \mid m \in \Delta_{\mathrm{GC}} \cap\left(\mathfrak{t}_{\mathrm{GC}}\right)_{\mathbb{Z}}^{*}\right\}$ of the space of holomorphic sections $H^{0}\left(L_{\text {symp }}^{\mathbb{F}}, \bar{\partial}^{s}\right)$ for all $s \in\left[0, s_{0}\right]$, which depends continuously on $s$. Thus, we have defined the basis $\left\{\sigma_{s}^{m} \mid m \in \Delta_{\mathrm{GC}} \cap\left(\mathfrak{t}_{\mathrm{GC}}\right)_{\mathbb{Z}}^{*}\right\}$ of the space of holomorphic sections $H^{0}\left(L_{\text {symp }}^{\mathbb{F}}, \bar{\partial}^{s}\right)$ for all $s \geq 0$.

7.3. Another gradient-Hamiltonian flow. To prove that the holomorphic sections defined by (7.6) converge to delta-function sections, we introduce another gradient-Hamiltonian flow.

Let us consider the family of varieties $f:\left(M_{n}(\mathbb{C}) \times \mathbb{C}\right) / / B \rightarrow \mathbb{C}$ constructed in Section 3.1. Put the standard Kähler metric on $\mathbb{C}$. Consider the map $F:\left(M_{n}(\mathbb{C}) \times \mathbb{C}\right) / / B \rightarrow \mathbb{P}_{\text {symp }} \times \mathbb{C}$ given by $F(x)=\left(\rho_{t, 0}(x), t\right)$ if $x \in V_{t, \text { symp }}=f^{-1}(t)$. We put the Kähler metric on the smooth part of $\left(M_{n}(\mathbb{C}) \times \mathbb{C}\right) / / B$ by pulling back the metric on $\mathbb{P}_{\text {symp }} \times \mathbb{C}$ by the map $F$. Consider the gradient-Hamiltonian flow along the straight-line path from 1 to 0 in $\mathbb{C}$. Since $V_{t, \text { symp }}$ are smooth manifolds for all $t \in(0,1]$, by Lemma 4.1 the vector field and thus the flow are defined on all of $V_{t \text {,symp }}$ for each $t \in(0,1]$, and also on $V_{0, \text { symp }}^{\circ}$, where $V_{0, \text { symp }}^{\circ}$ is the same as in (7.3). Let $V_{t, \text { symp }}^{\circ} \subset V_{t \text {,symp }}$ denote the image of $V_{0, \text { symp }}^{\circ}$ under the reverse flow. Then, 
due to Propositions 4.2 and 4.3, we have the following symplectic diffeomorphism and its lift defined by the gradient-Hamiltonian flow for $t \in[0,1]$ :

$$
\begin{array}{ccc}
\left.\left(L_{\text {symp }}^{V_{t}}, h^{V_{t}}, \nabla^{V_{t}}\right)\right|_{V_{t, \text { symp }}^{\circ}} & \stackrel{\tilde{\Phi}_{t}}{\longrightarrow} & \left.\left(L_{\text {symp }}^{V_{0}}, h^{V_{0}}, \nabla^{V_{0}}\right)\right|_{V_{0, \text { symp }}^{\circ}} \\
\downarrow & \downarrow \\
\left(V_{t, \text { symp }}^{\circ}, \rho_{t, 0}^{*} \omega_{\mathbb{P}}\right) & \stackrel{\Phi_{t}}{\longrightarrow} & \left(V_{0, \text { symp }}^{\circ}, \rho_{0,0}^{*} \omega_{\mathbb{P}}\right) .
\end{array}
$$

Let $\mu_{T_{\mathbb{P}}}: \mathbb{P}_{\text {symp }} \rightarrow \mathfrak{t}_{\mathbb{P}}^{*}$ be the moment map for the $T_{\mathbb{P}}$-action on $\left(\mathbb{P}_{\text {symp }}, \omega_{\mathbb{P}}\right)$. Set $\mu_{T_{\mathrm{GC}}}=\iota_{\mathrm{GC}}^{*} \circ \mu_{T_{\mathbb{P}}}: \mathbb{P}_{\text {symp }} \rightarrow \mathfrak{t}_{\mathrm{GC}}^{*}$. Fix an open set $B \subset \operatorname{Int} \Delta_{\mathrm{GC}}$ such that Int $\Delta_{\mathrm{GC}} \cap\left(\mathfrak{t}_{\mathrm{GC}}\right)_{\mathbb{Z}}^{*} \subset B$ and $\bar{B} \subset$ Int $\Delta_{\mathrm{GC}}$. Set $U_{0}=\mu_{T_{\mathrm{GC}}}^{-1}(B) \cap V_{0, \text { symp }} \subset$

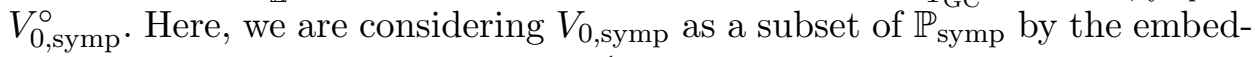
$\operatorname{ding} \rho_{0,0}$. Moreover, we set $U_{t}=\Phi_{t}^{-1}\left(U_{0}\right)$ and $U_{t}^{c}=V_{t, \text { symp }} \backslash U_{t}$. We denote the closure of $U_{t}$ in $V_{t \text {,symp }}$ by $\overline{U_{t}}$. Note that $\overline{U_{t}}$ and $U_{t}^{c}$ are compact.

Let $d_{\mathbb{P}}($,$) be the distance on \mathbb{P}$. Then, we have the following.

Lemma 7.1. For an arbitrary $\epsilon>0$, there exists $t_{1}>0$ such that $d_{\mathbb{P}}\left(\rho_{t, 0}(x), \rho_{0,0}\left(\Phi_{t}(x)\right)\right)<\epsilon$ for any $0 \leq t \leq t_{1}$ and $x \in \overline{U_{t}} \subset V_{t, \mathrm{symp}}$.

Proof. Fix an arbitrary small $t_{1}^{\prime}>0$. Then, $\overline{U_{t}}$ consists of regular points of $f:\left(M_{n}(\mathbb{C}) \times \mathbb{C}\right) / / B \rightarrow \mathbb{C}$ for any $0 \leq t \leq t_{1}^{\prime}$ and $\bigcup_{0 \leq t \leq t_{1}} \overline{U_{t}}$ is compact. As noted in Lemma 4.1, $|\operatorname{grad}(\Re f)|$ is non-zero at regular points of $f$. Therefore, there exists $c>0$ such that $|\operatorname{grad}(\Re f)| \geq c$ on $\overline{U_{t}}$, for every $t \in\left[0, t_{1}^{\prime}\right]$. Thus, the gradient-Hamiltonian vector field $Z$ satisfies $|Z| \leq \frac{1}{c}$ on $\overline{U_{t}}$ for $t \in\left[0, t_{1}^{\prime}\right]$. Since $\Phi_{t}$ is the flow of $Z$ over a "time" $t$, we finish the proof.

Similarly, we have the following.

Lemma 7.2. For an arbitrary $\epsilon>0$, there exists $t_{2}>0$ such that $d_{\mathbb{P}}\left(\rho_{1,0}\left(\Psi_{t}^{-1}(x)\right), \rho_{1,0}\left(\Psi_{0}^{-1} \circ \Phi_{t}(x)\right)\right)<\epsilon$ for all $0 \leq t \leq t_{2}$ and $x \in \overline{U_{t}} \subset$ $V_{t, \text { symp }}$, where $\Psi_{t}$ is the map in (7.2) or (7.3).

Proof. This follows from "smoothness in initial conditions" results in the theory of differential equations. Because the path $\gamma_{t}$ is close to the path $\gamma_{0}$ considered in Section 7.1 for small $t>0$, the resulting diffeomorphisms $\Psi_{t}$ and $\Psi_{0}$ are very close. Combining with Lemma 7.1 , we finish the proof.

7.4. Convergence to delta-function sections. For an $m \in \operatorname{Int} \Delta_{\mathrm{GC}} \cap$ $\left(\mathfrak{t}_{\mathrm{GC}}\right)_{\mathbb{Z}}^{*}$, we have chosen $\tilde{m} \in \Delta_{\mathbb{P}} \cap\left(\mathfrak{t}_{\mathbb{P}}\right)_{\mathbb{Z}}^{*}$ such that $\iota^{*}(\tilde{m})=m$ and defined the holomorphic section $\sigma_{s}^{m}$ by (7.6). From now on, we prove that, if we choose $t(s)$ appropriately for $s \geq 0$, the section $\frac{\sigma_{s}^{m}}{\left\|\sigma_{s}^{m}\right\|_{L^{1}\left(\mathbb{F}_{\mathrm{symp}}\right)}}$ converges to a delta-function section supported on the Bohr-Sommerfeld fiber $\mu_{\mathrm{GC}}^{-1}(m)$ as $s$ goes to infinity. Set, for $0 \leq t \leq 1, s>>0$,

$$
\tau_{t, s}^{m}=\frac{\underline{\tilde{\chi}}_{t, s}^{*}\left(\tilde{\rho}_{t, \mathrm{comp}}^{*} \sigma^{\tilde{m}}\right)}{\left\|\underline{\tilde{\chi}}_{t, s}^{*}\left(\tilde{\rho}_{t, \mathrm{comp}}^{*} \sigma^{\tilde{m}}\right)\right\|_{L^{1}\left(V_{t, \mathrm{symp}}\right)}} \in H^{0}\left(L_{\mathrm{symp}}^{V_{t}}, \underline{\tilde{\chi}}_{t, s}^{*} \bar{\partial}^{V_{t}}\right) .
$$


Since

$$
\tilde{\Psi}_{t}^{*} \tau_{t, s}^{m}=\frac{\tilde{\Psi}_{t}^{*} \underline{\tilde{\chi}}_{t, s}^{*}\left(\tilde{\rho}_{t, \mathrm{comp}}^{*} \sigma^{\tilde{m}}\right)}{\left\|\underline{\tilde{\chi}}_{t, s}^{*}\left(\tilde{\rho}_{t, \mathrm{comp}}^{*} \sigma^{\tilde{m}}\right)\right\|_{L^{1}\left(V_{t, \mathrm{symp}}\right)}}=\frac{\tilde{\Psi}_{t}^{*} \underline{\tilde{\chi}}_{t, s}^{*}\left(\tilde{\rho}_{t, \mathrm{comp}}^{*} \sigma^{\tilde{m}}\right)}{\left\|\tilde{\Psi}_{t}^{*} \tilde{\tilde{\chi}}_{t, s}^{*}\left(\tilde{\rho}_{t, \mathrm{comp}}^{*} \sigma^{\tilde{m}}\right)\right\|_{L^{1}\left(\mathbb{F}_{\mathrm{symp}}\right)}},
$$

we have $\tilde{\Psi}_{t(s)}^{*} \tau_{t(s), s}^{m}=\frac{\sigma_{s}^{m}}{\left\|\sigma_{s}^{m}\right\|_{L^{1}\left(\mathbb{F}_{\mathrm{symp}}\right)}}$, where $t(s)$ will be defined in Lemma 7.5 below.

For a section $\phi \in \Gamma\left(\left(L_{\text {symp }}^{\mathbb{F}}\right)^{*}\right)$, we denote the push-forward of $\phi$ with respect to the map $\tilde{\Psi}_{t}$ by $\tilde{\Psi}_{t *} \phi$, which is a section of the line bundle $\left(L_{\text {symp }}^{V_{t}}\right)^{*}$ for $t>0$ or a section of $\left(L_{\text {symp }}^{V_{0}}\right)^{*}$ restricted to some open dense subset of $V_{0 \text {,symp }}$ for $t=0$. In what follows, we omit the notation for the volume form when integrating on $\mathbb{F}_{\text {symp }}$ or $V_{t, \text { symp }}$, since it is preserved by the maps $\Psi_{t}$ and $\Phi_{t}$. First, we have the following:

Lemma 7.3. (1) For $m \in \operatorname{Int} \Delta_{\mathrm{GC}}, \mu_{T_{\mathrm{GC}}^{-1}}(m) \cap V_{0, \text { symp }}$ is a Bohr-Sommerfeld fiber for $\left(L_{\mathrm{symp}}^{V_{0}}, h^{V_{0}}, \nabla^{V_{0}}\right)$ if and only if $m \in\left(\mathfrak{t}_{\mathrm{GC}}\right)_{\mathbb{Z}}^{*}$.

(2) For $m \in \operatorname{Int} \Delta_{\mathrm{GC}} \cap\left(\mathfrak{t}_{\mathrm{GC}}\right)_{\mathbb{Z}}^{*}$, there exists a covariantly constant section $\delta_{m}$ of $\left.\left(L_{\mathrm{symp}}^{V_{0}}, h^{V_{0}}, \nabla^{V_{0}}\right)\right|_{\mu_{T_{\mathrm{GC}}}^{-1}(m) \cap V_{0, \mathrm{symp}}}$ and a measure $d \theta_{m}$ on $\mu_{T_{\mathrm{GC}}}^{-1}(m) \cap V_{0, \mathrm{symp}}$ which satisfy the following: for any $\phi \in \Gamma\left(\left(L_{\text {symp }}^{\mathbb{F}}\right)^{*}\right)$, there exists $C_{1}(s, \phi)>0$ for $s \geq 0$, such that $\lim _{s \rightarrow \infty} C_{1}(s, \phi)=0$ and

$$
\left|\int_{V_{0, \mathrm{symp}}}\left\langle\tilde{\Psi}_{0 *} \phi, \tau_{0, s}^{m}\right\rangle-\int_{\mu_{T_{\mathrm{GC}}}^{-1}(m) \cap V_{0, \mathrm{symp}}}\left\langle\tilde{\Psi}_{0 *} \phi, \delta_{m}\right\rangle d \theta_{m}\right| \leq C_{1}(s, \phi) .
$$

Proof. (1) follows from Proposition 6.6 (2).

(2) follows from Proposition 6.6 (4). Since the number of points in Int $\Delta_{\mathrm{GC}} \cap$ $\left(\mathfrak{t}_{\mathrm{GC}}\right)_{\mathbb{Z}}^{*}$ is finite, we can choose $C_{1}(s, \phi)$ independently of $m \in \operatorname{Int} \Delta_{\mathrm{GC}} \cap$ $\left(\mathfrak{t}_{\mathrm{GC}}\right)_{\mathbb{Z}}^{*}$

We take $U_{t} \subset V_{t \text {,symp }}$ as in Section 7.3. Then, we have the following.

Lemma 7.4. For each section $\phi \in \Gamma\left(\left(L_{\text {symp }}^{\mathbb{F}}\right)^{*}\right)$, the following holds:

$$
\begin{aligned}
& \left|\int_{\mathbb{F}_{\mathrm{symp}}}\left\langle\phi, \tilde{\Psi}_{t}^{*} \tau_{t, s}^{m}\right\rangle-\int_{\mu_{T_{\mathrm{GC}}}^{-1}(m) \cap V_{0, \mathrm{symp}}}\left\langle\tilde{\Psi}_{0 *} \phi, \delta_{m}\right\rangle d \theta_{m}\right| \\
& \leq C_{1}(s, \phi)+\operatorname{vol}\left(\mathbb{F}_{\mathrm{symp}}\right)\|\phi\|_{C^{0}\left(\mathbb{F}_{\mathrm{symp}}\right)}\left(\left\|\tau_{t, s}^{m}\right\|_{C^{0}\left(U_{t}^{c}\right)}+\left\|\tilde{\Phi}_{t}^{*} \tau_{0, s}^{m}\right\|_{C^{0}\left(U_{t}^{c}\right)}\right) \\
& \quad+\operatorname{vol}\left(\mathbb{F}_{\mathrm{symp}}\right)\|\phi\|_{C^{0}\left(\mathbb{F}_{\mathrm{symp}}\right)}\left\|\tau_{t, s}^{m}-\tilde{\Phi}_{t}^{*} \tau_{0, s}^{m}\right\|_{C^{0}\left(U_{t}\right)} \\
& \quad+\left\|\tilde{\Psi}_{t *} \phi-\tilde{\Phi}_{t}^{*} \tilde{\Psi}_{0 *} \phi\right\|_{C^{0}\left(U_{t}\right)} .
\end{aligned}
$$


Proof. Fix arbitrary $\phi \in \Gamma\left(\left(L_{\text {symp }}^{\mathbb{F}}\right)^{*}\right)$. Then, we have

$$
\begin{aligned}
& \left|\int_{\mathbb{F}_{\mathrm{symp}}}\left\langle\phi, \tilde{\Psi}_{t}^{*} \tau_{t, s}^{m}\right\rangle-\int_{\mu_{T_{\mathrm{GC}}}^{-1}(m) \cap V_{0, \mathrm{symp}}}\left\langle\tilde{\Psi}_{0 *} \phi, \delta_{m}\right\rangle d \theta_{m}\right| \\
& =\left|\int_{V_{t, \mathrm{symp}}}\left\langle\tilde{\Psi}_{t *} \phi, \tau_{t, s}^{m}\right\rangle-\int_{\mu_{T_{\mathrm{GC}}}^{-1}(m) \cap V_{0, \mathrm{symp}}}\left\langle\tilde{\Psi}_{0 *} \phi, \delta_{m}\right\rangle d \theta_{m}\right| \\
& \leq\left|\int_{V_{t, \mathrm{symp}}}\left\langle\tilde{\Psi}_{t *} \phi, \tau_{t, s}^{m}\right\rangle-\int_{V_{0, \mathrm{symp}}}\left\langle\tilde{\Psi}_{0 *} \phi, \tau_{0, s}^{m}\right\rangle\right| \\
& \quad+\left|\int_{V_{0, \mathrm{symp}}}\left\langle\tilde{\Psi}_{0 *} \phi, \tau_{0, s}^{m}\right\rangle-\int_{\mu_{T_{\mathrm{GC}}}^{-1}(m) \cap V_{0, \mathrm{symp}}}\left\langle\tilde{\Psi}_{0 *} \phi, \delta_{m}\right\rangle d \theta_{m}\right| .
\end{aligned}
$$

The second term on the right-hand side of (7.8) is estimated by (7.7). Next, we estimate the first term on the right-hand side of (7.8)

$$
\begin{aligned}
& \left|\int_{V_{t, \mathrm{symp}}}\left\langle\tilde{\Psi}_{t *} \phi, \tau_{t, s}^{m}\right\rangle-\int_{V_{0, \mathrm{symp}}}\left\langle\tilde{\Psi}_{0 *} \phi, \tau_{0, s}^{m}\right\rangle\right| \\
& =\left|\int_{V_{t, \mathrm{symp}}}\left\langle\tilde{\Psi}_{t *} \phi, \tau_{t, s}^{m}\right\rangle-\int_{V_{t, \mathrm{symp}}}\left\langle\tilde{\Phi}_{t}^{*} \tilde{\Psi}_{0 *} \phi, \tilde{\Phi}_{t}^{*} \tau_{0, s}^{m}\right\rangle\right| \\
& \leq\left|\int_{U_{t}}\left\langle\tilde{\Psi}_{t *} \phi, \tau_{t, s}^{m}\right\rangle-\left\langle\tilde{\Phi}_{t}^{*} \tilde{\Psi}_{0 *} \phi, \tilde{\Phi}_{t}^{*} \tau_{0, s}^{m}\right\rangle\right| \\
& \quad+\left|\int_{U_{t}^{c}}\left\langle\tilde{\Psi}_{t *} \phi, \tau_{t, s}^{m}\right\rangle-\left\langle\tilde{\Phi}_{t}^{*} \tilde{\Psi}_{0 *} \phi, \tilde{\Phi}_{t}^{*} \tau_{0, s}^{m}\right\rangle\right| \\
& \leq\left|\int_{U_{t}}\left\langle\tilde{\Psi}_{t *} \phi, \tau_{t, s}^{m}\right\rangle-\left\langle\tilde{\Phi}_{t}^{*} \tilde{\Psi}_{0 *} \phi, \tilde{\Phi}_{t}^{*} \tau_{0, s}^{m}\right\rangle\right| \\
& \quad+\operatorname{vol}\left(\mathbb{F}_{\mathrm{symp}}\right)\|\phi\|_{C^{0}\left(\mathbb{F}_{\mathrm{symp}}\right)}\left(\left\|\tau_{t, s}^{m}\right\|_{C^{0}\left(U_{t}^{c}\right)}+\left\|\tilde{\Phi}_{t}^{*} \tau_{0, s}^{m}\right\|_{C^{0}\left(U_{t}^{c}\right)}\right) .
\end{aligned}
$$

Finally, we estimate the first term on the right-hand side of (7.9). If we note that

$$
\begin{aligned}
& \int_{U_{t}}\left|\tilde{\Psi}_{t *} \phi\right| \leq \operatorname{vol}\left(U_{t}\right)\left\|\tilde{\Psi}_{t *} \phi\right\|_{C^{0}\left(U_{t}\right)} \leq \operatorname{vol}\left(\mathbb{F}_{\text {symp }}\right)\|\phi\|_{C^{0}\left(\mathbb{F}_{\text {symp }}\right)} \\
& \int_{U_{t}}\left|\tilde{\Phi}_{t}^{*} \tau_{0, s}^{m}\right|=\int_{U_{0}}\left|\tau_{0, s}^{m}\right| \leq \int_{U_{0}} \frac{\left|\underline{\tilde{\chi}}_{0, s}^{*}\left(\tilde{\rho}_{0, \mathrm{comp}}^{*} \sigma^{\tilde{m}}\right)\right|}{\left.\| \tilde{\rho}_{0, \mathrm{comp}}^{*} \sigma^{\tilde{m}}\right) \|_{L^{1}\left(V_{0, \mathrm{symp}}\right)}} \leq 1,
\end{aligned}
$$


then we have

$$
\begin{aligned}
& \left|\int_{U_{t}}\left\langle\tilde{\Psi}_{t *} \phi, \tau_{t, s}^{m}\right\rangle-\left\langle\tilde{\Phi}_{t}^{*} \tilde{\Psi}_{0 *} \phi, \tilde{\Phi}_{t}^{*} \tau_{0, s}^{m}\right\rangle\right| \\
& \quad \leq\left|\int_{U_{t}}\left\langle\tilde{\Psi}_{t *} \phi, \tau_{t, s}^{m}-\tilde{\Phi}_{t}^{*} \tau_{0, s}^{m}\right\rangle\right|+\left|\int_{U_{t}}\left\langle\tilde{\Psi}_{t *} \phi-\tilde{\Phi}_{t}^{*} \tilde{\Psi}_{0 *} \phi, \tilde{\Phi}_{t}^{*} \tau_{0, s}^{m}\right\rangle\right| \\
& \quad \leq\left\|\tau_{t, s}^{m}-\tilde{\Phi}_{t}^{*} \tau_{0, s}^{m}\right\|_{C^{0}\left(U_{t}\right)} \int_{U_{t}}\left|\tilde{\Psi}_{t *} \phi\right|+\left\|\tilde{\Psi}_{t *} \phi-\tilde{\Phi}_{t}^{*} \tilde{\Psi}_{0 *} \phi\right\|_{C^{0}\left(U_{t}\right)} \int_{U_{t}}\left|\tilde{\Phi}_{t}^{*} \tau_{0, s}^{m}\right| \\
& \quad \leq\left\|\tau_{t, s}^{m}-\tilde{\Phi}_{t}^{*} \tau_{0, s}^{m}\right\|_{C^{0}\left(U_{t}\right)} \operatorname{vol}\left(\mathbb{F}_{\mathrm{symp}}\right)\|\phi\|_{C^{0}\left(\mathbb{F}_{\mathrm{symp}}\right)}+\left\|\tilde{\Psi}_{t *} \phi-\tilde{\Phi}_{t}^{*} \tilde{\Psi}_{0 *} \phi\right\|_{C^{0}\left(U_{t}\right)} .
\end{aligned}
$$

By (7.7)-(7.10), we finish the proof of Lemma 7.4.

Next, we introduce a function $t:[0, \infty) \rightarrow \mathbb{R}$ so that the holomorphic sections $\sigma_{s}^{m}$ converges to delta-function sections as $s$ goes to infinity.

Lemma 7.5. There exists a strictly decreasing $C^{\infty}$-function $t:[0, \infty) \rightarrow$ $\mathbb{R}_{>0}$, where $\mathbb{R}_{>0}$ is the set of positive real numbers, with $t(0)=1$ and $\lim _{s \rightarrow \infty} t(s)=0$ which satisfies the following: for any $\phi \in \Gamma\left(\left(L_{\mathrm{symp}}^{\mathbb{F}}\right)^{*}\right)$, there exists a constant $C_{2}(s, \phi)>0$ with $\lim _{s \rightarrow \infty} C_{2}(s, \phi)=0$ such that

$$
\left|\int_{\mathbb{F}_{\mathrm{symp}}}\left\langle\phi, \tilde{\Psi}_{t(s)}^{*} \tau_{t(s), s}^{m}\right\rangle-\int_{\mu_{T_{\mathrm{GC}}}^{-1}(m) \cap V_{0, \mathrm{symp}}}\left\langle\tilde{\Psi}_{0 *} \phi, \delta_{m}\right\rangle d \theta_{m}\right| \leq C_{2}(s, \phi) .
$$

Proof. First, we estimate the term $\left\|\tilde{\Phi}_{t}^{*} \tau_{0, s}^{m}\right\|_{C^{0}\left(U_{t}^{c}\right)}$ in Lemma 7.4. Due to Proposition $6.6(3)$, there exists $C_{3}(s)>0$ such that $\lim _{s \rightarrow \infty} C_{3}(s)=0$ and, for any $t>0$,

$$
\left\|\tilde{\Phi}_{t}^{*} \tau_{0, s}^{m}\right\|_{C^{0}\left(U_{t}^{c}\right)}=\left\|\tau_{0, s}^{m}\right\|_{C^{0}\left(U_{0}^{c}\right)} \leq C_{3}(s) .
$$

Next, we estimate other terms in Lemma 7.4. In Section 7.3, we fixed an open set $B \subset \operatorname{Int} \Delta_{\mathrm{GC}}$ such that Int $\Delta_{\mathrm{GC}} \cap\left(\mathfrak{t}_{\mathrm{GC}}\right)_{\mathbb{Z}}^{*} \subset B$ and $\bar{B} \subset \operatorname{Int} \Delta_{\mathrm{GC}}$. We set $U_{0}=\mu_{T_{\mathrm{GC}}}^{-1}(B) \cap V_{0 \text {,symp }} \subset V_{0, \text { symp }}^{\circ}$ and $U_{t}=\Phi_{t}^{-1}\left(U_{0}\right)$. Now, we also take an open set $B_{1} \subset \operatorname{Int} \Delta_{\mathrm{GC}}$ such that $\operatorname{Int} \Delta_{\mathrm{GC}} \cap\left(\mathfrak{t}_{\mathrm{GC}}\right)_{\mathbb{Z}}^{*} \subset B_{1}$ and $\overline{B_{1}} \subset B$. Then, due to Proposition 6.6 (3), there exists $C_{4}(s)>0$ such that $\lim _{s \rightarrow \infty} C_{4}(s)=0$ and, for any $s \geq 0$ and $m \in \operatorname{Int} \Delta_{\mathrm{GC}} \cap\left(\mathfrak{t}_{\mathrm{GC}}\right)_{\mathbb{Z}}^{*}$,

$$
\left\|\frac{\tilde{\chi}_{s}^{*} \sigma^{\tilde{m}}}{\left\|\left.\tilde{\chi}_{s}^{*} \sigma^{\tilde{m}}\right|_{V_{0, \text { symp }}}\right\|_{L^{1}\left(V_{0, \mathrm{symp}}\right)}}\right\|_{C^{0}\left(\mathbb{P}_{\mathrm{symp}} \backslash \mu_{T_{\mathrm{GC}}}^{-1}\left(B_{1}\right)\right)} \leq C_{4}(s) .
$$

Since $\rho_{0, s}=\rho_{0,0}: V_{0, \text { symp }} \rightarrow \mathbb{P}_{\text {symp }}$ for $s \geq 0$ by Proposition $6.5(2)$, we have

$$
\rho_{0, s}\left(U_{0}^{c}\right)=\rho_{0,0}\left(U_{0}^{c}\right) \subset \mathbb{P}_{\text {symp }} \backslash \mu_{T_{\mathrm{GC}}}^{-1}\left(B_{1}\right)
$$


Note that

$$
\lim _{t \rightarrow 0}\left\|\underline{\tilde{\chi}}_{t, s}^{*}\left(\tilde{\rho}_{t, \mathrm{comp}}^{*} \sigma^{\tilde{m}}\right)\right\|_{L^{1}\left(V_{t, \mathrm{symp}}\right)}=\left\|\underline{\tilde{\chi}}_{0, s}^{*}\left(\tilde{\rho}_{0, \mathrm{comp}}^{*} \sigma^{\tilde{m}}\right)\right\|_{L^{1}\left(V_{0, \mathrm{symp}}\right)} \neq 0 .
$$

Since $U_{t}^{c}$ is compact, for each $n=1,2, \ldots$, there exists $t_{n} \in(0,1]$ which is independent of $\phi$ and satisfies the following (7.13) holds for each $s \in[n, n+1]$ and $t \in\left[0, t_{n}\right]$ :

$$
\rho_{t, s}\left(U_{t}^{c}\right) \subset \mathbb{P}_{\mathrm{symp}} \backslash \mu_{T_{\mathrm{GC}}}^{-1}\left(B_{1}\right), \frac{\left\|\underline{\tilde{\chi}}_{t, s}^{*}\left(\tilde{\rho}_{t, \mathrm{comp}}^{*} \sigma^{\tilde{m}}\right)\right\|_{L^{1}\left(V_{t, \mathrm{symp}}\right)}}{\left\|\underline{\tilde{\chi}}_{0, s}^{*}\left(\tilde{\rho}_{0, \mathrm{comp}}^{*} \sigma^{\tilde{m}}\right)\right\|_{L^{1}\left(V_{0, \mathrm{symp}}\right)}} \geq \frac{1}{2} .
$$

By (7.12) and (7.13), we have, for each $s \in[n, n+1]$ and $t \in\left[0, t_{n}\right]$

$$
\begin{aligned}
& C_{4}(s) \geq\left\|\frac{\tilde{\rho}_{t, s}^{*}\left(\tilde{\chi}_{s}^{*} \sigma^{\tilde{m}}\right)}{\left\|\underline{\tilde{\rho}}_{0, s}^{*}\left(\tilde{\chi}_{s}^{*} \sigma^{\tilde{m}}\right)\right\|_{L^{1}\left(V_{0, \mathrm{symp}}\right)}}\right\|_{C^{0}\left(U_{t}^{c}\right)} \\
& =\left\|\frac{\underline{\tilde{x}}_{t, s}^{*}\left(\tilde{\rho}_{t, \mathrm{comp}}^{*} \sigma^{\tilde{m}}\right)}{\left\|\underline{\tilde{\chi}}_{0, s}^{*}\left(\tilde{\rho}_{0, \mathrm{comp}}^{*} \sigma^{\tilde{m}}\right)\right\|_{L^{1}\left(V_{0, \mathrm{symp}}\right)}}\right\|_{C^{0}\left(U_{t}^{c}\right)}
\end{aligned}
$$

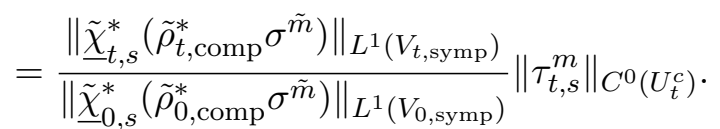

By (7.13) and (7.14), we have, for each $s \in[n, n+1]$ and $t \in\left[0, t_{n}\right]$

$$
\left\|\tau_{t, s}^{m}\right\|_{C^{0}\left(U_{t}^{c}\right)} \leq 2 C_{4}(s)
$$

Moreover, due to Lemmas 7.1 and 7.2, taking smaller $t_{n}>0$ if necessary, we may also conclude that the following (7.16) and (7.17) hold for each $s \in[n, n+1]$ and $t \in\left[0, t_{n}\right]:$

$$
\begin{aligned}
& \left\|\tau_{t, s}^{m}-\tilde{\Phi}_{t}^{*} \tau_{0, s}^{m}\right\|_{C^{0}\left(U_{t}\right)} \leq \frac{1}{n+2} \text { for any } m \in \operatorname{Int} \Delta \cap\left(\mathfrak{t}_{\mathrm{GC}}\right)_{\mathbb{Z}}^{*} \\
& \left\|\tilde{\Psi}_{t *} \phi-\tilde{\Phi}_{t}^{*} \tilde{\Psi}_{0 *} \phi\right\|_{C^{0}\left(U_{t}\right)} \leq \frac{\|\phi\|_{C^{1}\left(\mathbb{F}_{\mathrm{symp}}\right)}}{n+2} \text { for any } \phi \in \Gamma\left(\left(L_{\mathrm{symp}}^{\mathbb{F}}\right)^{*}\right) .
\end{aligned}
$$

By Lemma 7.4 together with (7.11), (7.15), (7.16) and (7.17), we have, for each section $\phi \in \Gamma\left(\left(L_{\text {symp }}^{\mathbb{F}}\right)^{*}\right), n=1,2, \ldots, s \in[n, n+1]$ and $t \in\left[0, t_{n}\right]$

$$
\begin{gathered}
\left|\int_{\mathbb{F}_{\mathrm{symp}}}\left\langle\phi, \tilde{\Psi}_{t}^{*} \tau_{t, s}^{m}\right\rangle-\int_{\mu_{T_{\mathrm{GC}}}^{-1}(m) \cap V_{0, \mathrm{symp}}}\left\langle\tilde{\Psi}_{0 *} \phi, \delta_{m}\right\rangle d \theta_{m}\right| \\
\leq C_{1}(s, \phi)+\operatorname{vol}\left(\mathbb{F}_{\mathrm{symp}}\right)\|\phi\|_{C^{0}\left(\mathbb{F}_{\mathrm{symp}}\right)}\left(2 C_{4}(s)+C_{3}(s)\right) \\
\quad+\operatorname{vol}\left(\mathbb{F}_{\mathrm{symp}}\right)\|\phi\|_{C^{0}\left(\mathbb{F}_{\mathrm{symp}}\right)} \frac{1}{s+1}+\frac{\|\phi\|_{C^{1}\left(\mathbb{F}_{\mathrm{symp}}\right)}}{s+1} .
\end{gathered}
$$


We can take a continuous decreasing function $t:[0, \infty) \rightarrow \mathbb{R}$ with $t(0)=1$ and $\lim _{s \rightarrow \infty} t(s)=0$ such that $t(n) \leq t_{n}$ for $n>0$. Thus, we finish the proof of Lemma 7.5.

We use $t(s)$ in Lemma 7.5 to define the complex structure $J_{s}$ by (7.5) and the holomorphic section $\sigma_{s}^{m}$ by (7.6). If we recall $\tilde{\Psi}_{t(s)}^{*} \tau_{t(s), s}^{m}=\frac{\sigma_{s}^{m}}{\left\|\sigma_{s}^{m}\right\|_{L^{1}\left(\mathbb{F}_{\mathrm{symp}}\right)}}$, then we have

$$
\lim _{s \rightarrow \infty} \int_{\mathbb{F}_{\mathrm{symp}}}\left\langle\phi, \frac{\sigma_{s}^{m}}{\left\|\sigma_{s}^{m}\right\|_{L^{1}\left(\mathbb{F}_{\mathrm{symp}}\right)}}\right\rangle=\int_{\mu_{T_{\mathrm{GC}}}^{-1}(m) \cap V_{0, \mathrm{symp}}}\left\langle\tilde{\Psi}_{0 *} \phi, \delta_{m}\right\rangle d \theta_{m} .
$$

Due to Corollary 3.3, if we define a covariantly constant section $\delta_{m}^{\mathbb{F}}$ of $\left.\left(L^{\mathbb{F}}, h^{\mathbb{F}}, \nabla^{\mathbb{F}}\right)\right|_{\mu_{\mathrm{GC}}^{-1}(m)}$ by pulling $\delta_{m}$ on $\mu_{T_{\mathrm{GC}}}^{-1}(m) \cap V_{0 \text {,symp }}$ back by $\tilde{\Psi}_{0}$, then we have the desired convergence in Theorem 2.1 (4).

\section{References}

[Ab1] M. Abreu, Kähler geometry of toric varieties and extremal metrics, Internat. J. Math. 6 (1998), 641-651.

[Ab2] M. Abreu, Kähler geometry of toric manifolds in symplectic coordinates, in 'Symplectic and contact topology: interactions and perspectives' (Y. Eliashberg, B. Khesin and F. Lalonde, eds.), Fields Institute Communications, Vol. 35, Amer. Math. Soc., Providence, 2003.

[AB] V. Alexeev and M. Brion, Toric degenerations of spherical varieties, Selecta Math. (N.S.) 10 (2004), 453-478.

[Au] M. Audin, Torus actions on symplectic manifolds, Birkhäuser, Basel, 2004.

[BFMN] T. Baier, C. Florentino, J.M. Mourão and J.P. Nunes, Toric Kähler metrics seen from infinity, quantization and compact tropical amoebas, J. Differential Geom. 89 (2011), 411-454.

[C] P. Caldero, Toric degenerations of Schubert varieties, Transform. Groups, 7 (2002), 51-60.

[D] T. Delzant, Hamiltoniens périodiques et image convexe de l'application moment, Bull. Soc. Math. France, 116 (1988), 315-339.

[F] W. Fulton, Introduction to toric varieties, Princeton University Press, Princeton, NJ, 1993.

[GL] N. Gonciulea and V. Lakshmibai, Degenerations of flag and Schubert varieties to toric varieties, Transform. Groups, 1 (1996), 215-248.

[Gu1] V. Guillemin, Kähler structures on toric varieties, J. Differential Geom. 40 (1994), 285-309.

[Gu2] V. Guillemin, Moment maps and combinatorial invariants of Hamiltonian $T^{N}$ spaces, Birkhäuser, Boston, 1994.

[GS] V. Guillemin and S. Sternberg, The Gel'fand-Cetlin system and quantization of the complex flag manifolds, J. Funct. Anal. 52 (1983), 106-128.

[JW] L. Jeffrey and J. Weitsman, Bohr-Sommerfeld orbits in the moduli space of flat connections and the Verlinde dimension formula, Commun. Math. Phys. 150 (1992), 593-630. 
[KM] M. Kogan and E. Miller, Toric degeneration of Schubert varieties and GelfandTsetlin polytopes, Adv. Math. 193 (2005), 1-17.

[NNU] T. Nishinou, Y. Nohara and K. Ueda, Toric degenerations of Gelfand-Cetlin systems and potential functions, Adv. Math. 224 (2010), 648-706.

[R] W.-D. Ruan, Lagrangian torus fibration of quintic hypersurfaces. I. Fermat quintic case, in 'Winter School on Mirror Symmetry, Vector Bundles and Lagrangian Submanifolds (Cambridge, MA, 1999)', AMS/IP Stud. Adv. Math., 23, 297-332, Amer. Math. Soc., Providence, 2001.

[S] J. Śniatycki, Geometric quantization and quantum mechanics, Applied Mathematical Sciences, Springer, New York, 1980.

[W] N.M.J. Woodhouse, Geometric quantization, 2nd ed., Oxford University Press, Oxford, 2007.

Department of Mathematics and Computer Science

Mount Allison University

67 York St, Sackville, NB E4L 1E6, Canada

E-mail address: mhamilton@mta.ca

Graduate School of Mathematical Sciences

UNIVERSITY OF TOKYO

3-8-1 Komaba, Meguro-Ku, Tokyo 153-8914, Japan

Current address: Department of Mathematics

School of Science and Technology

MEIJI UNIVERSITY

1-1-1 Higashi-Mita TAMA-KU, KaWASAKi 214-8571, JaPAN

E-mail address: konno@ms.u-tokyo.ac.jp

Current e-mail address: hkonno@meiji.ac.jp

Received 05/10/2011, accepted 08/09/2013

The first named author is supported by the JSPS Postdoctoral Fellowship for Foreign Researchers. The second named author is supported by the JSPS Grant-in-Aid for Scientific Research (C), numbers 19540067 and 23540072. The authors would like to thank Y. Nohara for discussions and the referee for a number of suggestions. 
